\title{
Regards croisés sur la station aurignacienne de Brignol (Villeneuve-sur-Lot, Lot-et-Garonne, France)
}

Approches taphonomique, pétroarchéologique, technoéconomique et technofonctionnelle de l'industrie lithique

Crossed perspectives at the Aurignacian site of Brignol (Villeneuve-sur-Lot, Lotet-Garonne, France) : taphonomic, petroarchaeological, technoeconomic, and technofunctional approaches to the lithic industry

Lars Anderson, Lorène Chesnaux, Mathieu Rué, Régis Picavet, Paul Fernandes, André Morala, Solène Caux, Pascal Tallet, Jean-Baptiste Caverne et Emmanuelle Kawalek

\section{(2) OpenEdition}

1 Journals

Édition électronique

URL : http://journals.openedition.org/paleo/3039

DOI : $10.4000 /$ paleo.3039

ISSN : 2101-0420

Éditeur

SAMRA

Édition imprimée

Date de publication : 30 décembre 2016

Pagination : 11-42

ISSN : $1145-3370$

Référence électronique

Lars Anderson, Lorène Chesnaux, Mathieu Rué, Régis Picavet, Paul Fernandes, André Morala, Solène Caux, Pascal Tallet, Jean-Baptiste Caverne et Emmanuelle Kawalek, «Regards croisés sur la station aurignacienne de Brignol (Villeneuve-sur-Lot, Lot-et-Garonne, France) », PALEO [En ligne], 27 | 2016, mis en ligne le 01 juin 2018, consulté le 07 juillet 2020. URL : http://journals.openedition.org/paleo/3039 ; DOI : https://doi.org/10.4000/paleo.3039 d'Utilisation Commerciale - Pas de Modification 4.0 International. 


\title{
Regards croisés sur la station aurignacienne de Brignol (Villeneuve-sur-Lot, Lot-et-Garonne, France) : approches taphonomique, pétroarchéologique, technoéconomique et technofonctionnelle de l'industrie lithique
}

\author{
Lars ANDERSON(a), Lorène CHESNAUX(b), Mathieu RUÉ(c), \\ Régis PICAVET ${ }^{(d)}$, Paul FERNANDES ${ }^{(e)}$, André MORALA ${ }^{(1)}$, Solène CAUX(g), \\ Pascal TALLET ${ }^{(\mathrm{h})}$, Jean-Baptiste CAVERNE ${ }^{(i)}$, Emmanuelle KAWALEK ${ }^{(j)}$
}

Résumé : Le site de Brignol fournit un nouvel ensemble archéologique à rattacher à un faciès très restreint de l'Aurignacien récent, celui à grattoirs Caminade. Représenté à l'heure actuelle de manière significative sur seulement huit sites du Sud-Ouest français, ce faciès reste jusqu'à aujourd'hui relativement énigmatique au sein du stade récent de la macro-culture aurignacienne. S'agit-il d'un faciès fonctionnel, complémentaire d'un autre faciès de cette même phase chronologique, ou bien au contraire d'une entité régionale et/ou chronologique distincte ? Plusieurs facteurs compliquent l'obtention aisée d'une réponse à cette question. Tout d'abord, des problèmes taphonomiques dans les sites stratifiés obscurcissent notre compréhension de la relation entre les grattoirs Caminade et les autres faciès de l'Aurignacien récent. D'autre part, l'absence, à ce jour, de remontages sur une telle série nous a empêchés de compléter le schéma opératoire existant de ce mode de production, ce qui est nécessaire pour comprendre son articulation avec d'autres modes de productions connues pour cette phase.

L'étude technoéconomique de la série lithique de Brignol, des remontages et leur croisement avec des analyses technofonctionnelles, pétroarchéologiques et taphonomiques, fournissent un nouveau jalon pour la compréhension de ce faciès particulier. La description du schéma opératoire de la production des grattoirs Caminade, les données tracéologiques et l'analyse des possibles fonctions du site permettent d'envisager de nouvelles hypothèses à explorer au sujet du fonctionnement de ces objets et plus largement sur le statut de ce faciès au sein de l'Aurignacien récent.

Mots-clés : Aurignacien récent, grattoir Caminade, géoarchéologie, pétroarchéologie, technologie lithique, chaîne opératoire, tracéologie.

(a) Université de Toulouse II Jean-Jaurès, 5 Allée Antonio Machado, FR-31058 Toulouse ; UMR 5608 CNRS TRACES lars.anderson@univ-tlse2.fr

(b) UMR 7041 CNRS ArScAn - Équipe ethnologie préhistorique, Maison Archéologie et Ethnologie, René-Ginouvès, 21 Allée de l'Université, FR-92000 Nanterre - chesnauxlorene@yahoo.fr

(c) Paléotime, 6173 rue J.-S. Achard-Picard, FR- 38250 Villard-de-Lans ; UMR 5140 CNRS, Archéologie des Sociétés Méditerranéennes - Université Paul-Valéry Montpellier 3, mathieu.rue@paleotime.fr

(d) Paléotime, 6173 rue J.-S. Achard-Picard, FR-38250 Villard-de-Lans ; UMR 7269 CNRS LAMPEA, Aix-Marseille Université regis.picavet@paleotime.fr

(e) Paléotime, 6173 rue J.-S. Achard-Picard, FR-38250 Villard-de-Lans ; UMR 5199 CNRS PACEA, Université Bordeaux 1, (f) Musée national de Préhistoire, FR-24620 Les Eyzies-de-Tayac ; UMR 5199 CNRS PACEA, Université Bordeaux 1 andre.morala@orange.fr

(g) UMR 5199 CNRS PACEA, Université de Bordeaux 1, Bâtiment B8 Allée Geoffroy Saint Hilaire, CS 50023, FR-33615 Pessac solene.caux@gmail.com

(h) Paléotime, 6173 rue J.-S. Achard-Picard, FR-38250 Villard-de-Lans - pascal.tallet@paleotime.fr

(i) Paléotime, 6173 rue J.-S. Achard-Picard, FR-38250 Villard-de-Lans - jean-baptiste.caverne@ paleotime.fr

(j) Indépendante - kawaemma@yahoo.fr 


\begin{abstract}
Crossed perspectives at the Aurignacian site of Brignol (Villeneuve-sur-Lot, Lot-et-Garonne, France): taphonomic, petroarchaeological, technoeconomic, and technofunctional approaches to the lithic industry. The site of Brignol provides a new archaeological assemblage attributable to the quite particular grattoir Caminade facies of the Recent Aurignacian. Identified in relatively important quantities on only eight sites to date, the role of this facies within the recent phase of the Aurignacian macro-cultural phenomenon remains quite enigmatic. Is it a functional facies, complimentary with other variants during this period, or can it be considered its own separate chronological and/or regional entity? Several factors impede our ability to provide an adequate answer to this question at this juncture. Firstly, taphonomic issues in stratified sites render our comprehension of the relationship between grattoirs Caminade and other chronological markers of the Recent Aurignacian less than clear, while the absence of refits on such a series to date has made it impossible to complete the existing description the grattoir Caminade operational sequence, which is in turn necessary to evaluate its compatibility with other modes of production present during this phase.

The technoeconomical study of the lithic assemblage from Brignol, systematic refits, combined with technofunctional, petroarchaeological, and taphonomic analyses, provide a new milestone for the comprehension of this particular archaeological facies. While Brignol cannot provide answers to all of our questions, the description of the operative schema of grattoir Caminade production, data from micro-wear analyses, as well as possible site functions, allow us to evoke novel hypotheses to explore regarding how these particular objets functioned and what the role of this facies is within the larger Recent Aurignacian period.
\end{abstract}

Key-words: Recent Aurignacian, grattoir Caminade, geoarchaeology, petroarchaeology, lithic technology, chaîne opératoire, use-wear analysis.

\section{1 - Introduction}

La fouille préventive du site de Brignol, situé à l'est de Villeneuve-sur-Lot, à environ $150 \mathrm{~m}$ de la rive droite du Lot (fig. 1a), a fourni un nouveau jalon pour la période récente du complexe aurignacien. Dans cet article, nous présentons l'analyse géoarchéologique, pétroarchéologique, technoéconomique et technofonctionelle de cette série attribuée à l'Aurignacien récent à grattoirs Caminade.

\section{1 - Identité d'un faciès industriel régional}

À l'image de l'ensemble de l'Aurignacien en Aquitaine, le stade récent de ce technocomplexe s'affirme par un grand nombre de sites, parmi lesquels celui de Brignol. Ce stade récent, relativement bien attesté dans le nord du Bassin aquitain, offre des spécificités typotechnologiques originales s'inscrivant dans un environnement chronoculturel riche, marqué, en conséquence, par une certaine complexité.

La composante industrielle de ce technocomplexe, qui comprend une fraction typologique assez homogène d'outils classiques du fonds commun, s'articule au sein des assemblages autour de quelques morphotypes de fréquence variable : burins busqués, burins des Vachons, burins carénés, lamelles Dufour (sous type Dufour et Rocde-Combes), lamelles Caminade, pointes de Font-Yves et grattoirs Caminade (e.g. Sonneville-Bordes et Mortureux 1955 ; Sonneville-Bordes 1960 ; Rigaud 1982 ; Lucas 1999 ; Bordes et Lenoble 2002 ; Morala, Lenoir et Turq 2005 ; Pesesse et Michel 2006 ; Pesesse 2008 ; Michel 2010). Cette complexité est vraisemblablement à la fois le résultat d'une variabilité chronologique et géographique, que nous avons du mal à distinguer en raison de l'ancienneté de la majorité des fouilles en question et d'un manque relatif de vérification de l'intégrité des ensembles suivant des méthodologies récentes (Bordes 2002). Au sein de cette complexité, ayant pour cause différents biais, se retrouve un outil-type particulier attribué dès sa reconnaissance à l'Aurignacien récent (Sonneville-Bordes et Mortureux 1955), le grattoir Caminade, auquel se rattache l'assemblage de Brignol que nous examinerons ici, mis en perspective dans son contexte culturel.

La mise en évidence dans le département du Lot-etGaronne de ce nouveau jalon industriel à grattoirs Caminade est un élément particulièrement important pour la compréhension du rôle de cet outil-type qui, pour l'heure, n'est attesté de manière significative que dans huit sites essentiellement dans le nord du Bassin aquitain (Brignol, Caminade, Grotte XVI, Le Flageolet, Las Pélénos, Maldidier, Le Pigeonnier et récemment mis en évidence (2016) à Fournol, dans le Lot, par A. Morala) (SonnevilleBordes et Mortureux 1955 ; Rigaud 1982 ; Quintard 1995 ; Lucas 2000 ; Morala, Lenoir et Turq 2005), et par des exemplaires isolés sur trois sites (La Grotte Dufour, Le Prissé, Brassempouy) qui semblent s'éloigner de la concentration principale (Demars et Laurent 1992 ; Michel 2010 ; D. Colonge, com. pers. ; F. Bon, com. pers.)

La particularité typologique qu'induit la présence des grattoirs Caminade s'affirme clairement comme un phénomène, à ce jour, très majoritairement aquitain. Elle a été reconnue par $\mathrm{D}$. de Sonneville-Bordes et $\mathrm{B}$. Mortureux sur le gisement de l'abri Caminade-Ouest (SonnevilleBordes et Mortureux 1955). Les chercheurs qui exploitèrent ce site observèrent, dans le niveau supérieur attribué à l'Aurignacien récent sur la base de la présence notamment de Burins busqués, l'existence d'outils microlithiques qu'ils dénommèrent par similitude morpho-typologique « grattoirs Caminade » (Sonneville-Bordes 1970). Ces microlithes sur éclat lamellaire ou courte lamelle ont été ainsi dénommés à cause de la retouche directe de la partie proximale qui évoque un front de grattoir (Sonneville-Bordes et Mortureux 

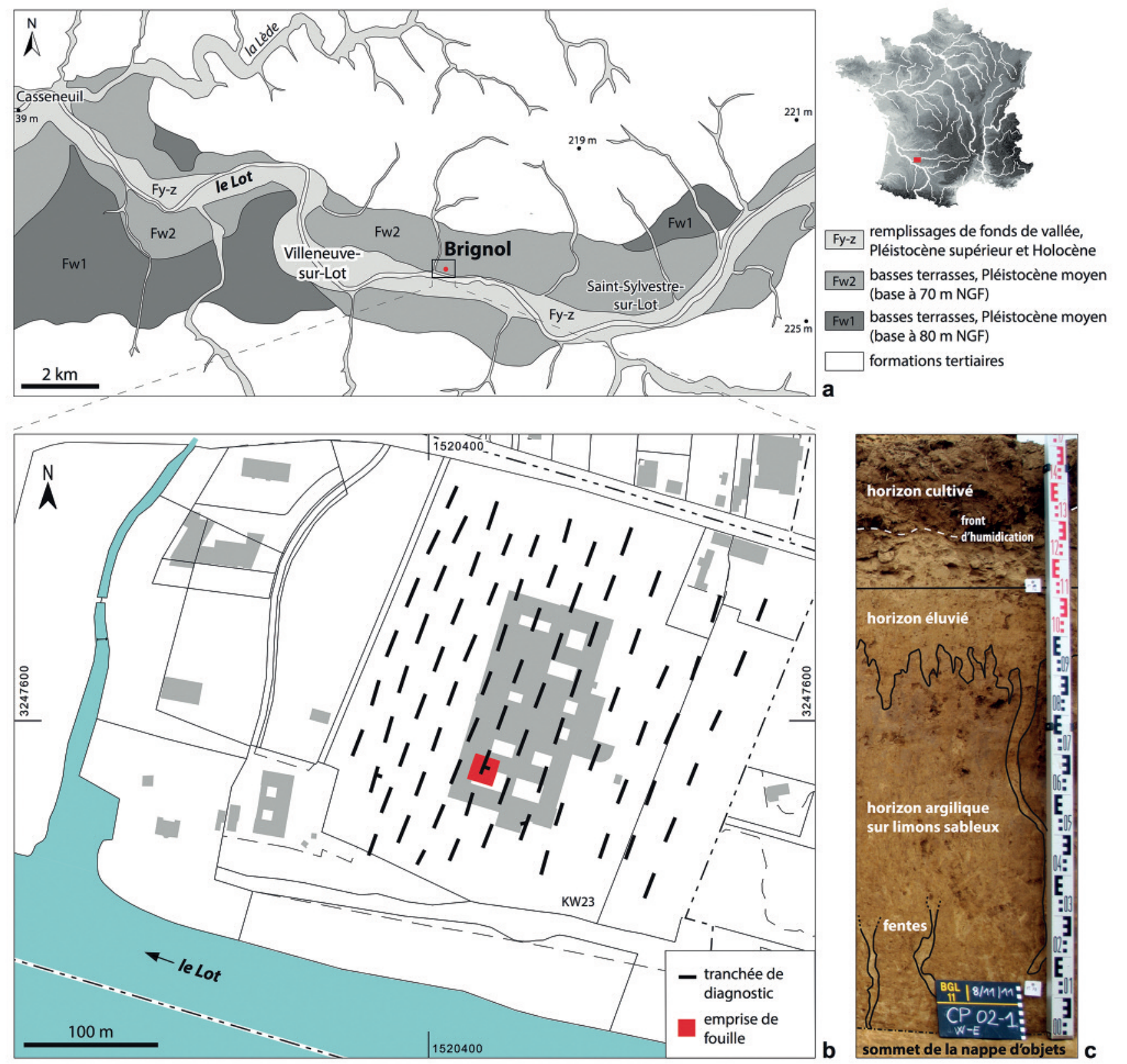

Figure 1 - Contexte géomorphologique et localisation du site (M. Rué). a. D'après la carte géologique à 1/50000 de Villeneuve-sur-Lot (Capdeville et Turq 1999). b. D'après le cadastre de 2016 et le plan du diagnostic (Ballarin et al. 2011). Coordonnées RGF93. c. Séquence stratigraphique de couverture du site (coupe 2.1).

Figure 1 - Geomorphological context and site localisation (M. Rué). a. According to 1/50000 scale geological map of Villeneuve-sur-Lot (Capdeville and Turq 1999). b. According to 2016 land registry and diagnostic intervention map (Ballarin et al. 2011). RGF93 coordinate system. c. Stratigraphic sequence of superposing sediments (profile 2.1)

1956), qui s'oppose souvent à une troncature inverse convexe en partie distale (Demars et Laurent 1992).

Dans ce niveau archéologique, les outils du fonds commun domestique accompagnent de nombreux burins busqués, grattoirs à museaux et grattoirs carénés, ainsi que des lamelles à retouche inverse, sous-type Dufour et Roc de Combe. Ce mélange de marqueurs chronologiques de l'Aurignacien est sans doute en grande partie le reflet des méthodes de fouilles de l'époque, qui n'intégraient pas les aspects géoarchéologiques à la compréhension de la formation des gisements. La reprise des fouilles à Caminade-Est (Bordes et Lenoble 2002) a permis la révision de la stratigraphie du site, mais malheureusement les grattoirs Caminade se trouvaient à Caminade-Ouest où une telle révision stratigraphique reste à effectuer. Une autre particularité d'intérêt est la présence, comme à Brignol, de galets façonnés et utilisés sur place (SonnevilleBordes et Mortureux 1955 - p. 37 ; Sonneville-Bordes 1970 - p. 110-111). 
Les prolongements de cette découverte se traduisirent par la mise en évidence dans quelques sites de la vallée de la Dordogne, de la Vézère et du Lot, d'assemblages industriels comportant en nombre variable ce type de « micrograttoir » : un peu plus de deux cents au Pigeonnier (Morala, Lenoir et Turq 2005), environ cent soixante-dix au Flageolet (Rigaud 1982 ; Lucas 2000), seize à la Grotte XVI (Rigaud 1982 ; Lucas 2000), environ cinq à Maldidier (Rigaud 1982 ; Lucas 2000), une dizaine à Las Pélénos 2 (Quintard 1995) et, à l'heure actuelle, huit à Fournol (A. Morala, com. pers.). À cette liste s'ajoutent des pièces isolées mentionnées ci-dessus trouvées à la Grotte Dufour (Demars et Laurent 1992, Michel 2010), au Prissé (D. Colonge, com. pers.) et à Brassempouy ( $F$. Bon, com. pers.).

\section{2 - Les enjeux}

II serait facile de croire que l'aire réduite dans laquelle se retrouve ce nombre restreint de sites principaux permettrait une compréhension à haute résolution de ce faciès très particulier de l'Aurignacien. Cette forte résolution aurait ainsi pu nous permettre d'opérer une distinction entre faciès fonctionnel ou faciès chronologique (Binford 1983). Malheureusement, les données ne sont pas aussi claires que l'on souhaiterait. II existe des associations entre grattoirs Caminade et d'autres marqueurs culturels plus classiques de l'Aurignacien récent, mais celles-ci se trouvent le plus souvent dans des sites stratifiés où une réévaluation de l'intégrité des couches archéologiques serait nécessaire. La précision des différentes phases de l'Aurignacien récent a permis de proposer d'intégrer les grattoirs Caminade dans la phase dite « Aurignacien récent classique à burins busqués " (Michel 2010). C'était notamment la réinterprétation de l'archéoséquence du Flageolet I (Michel 2010), qui a permis cette association. Pour le Flageolet I, nous notons tout de même la présence de grattoirs Caminade dans toute l'ancienne séquence (couches VIII, IX et IX ; Rigaud 1982 ; Lucas 2000) et dans presque l'intégralité de la séquence redéfinie (niveaux $B$, B', C, D et F ; Michel 2010). Pour l'abri Caminade une intégration semblable pourrait être avancée, mais ça nécessiterait la supposition que l'Aurignacien de la couche supérieur niveau $\mathrm{C}$ (contenant les grattoirs Caminade) (Sonneville-Bordes et Mortureux 1955) de CaminadeOuest correspond à l'Aurignacien récent plus récemment défini dans les couches D2i et D2s de Caminade-Est (Bordes et Lenoble 2001, 2002), la couche D2i livrant une date qui semble vieille, vers 39000 Cal BP (Rigaud 2001; Michel 2010). Ainsi, à l'heure actuelle, l'association entre les grattoirs Caminade et l'Aurignacien récent à burins busqués à l'abri Caminade reste assez ambiguë. Enfin, cette association n'a pas encore pu être confirmée sur les autres sites, suite à des problèmes taphonomiques, de méthodes de fouilles, ou encore sur certains gisements (le Pigeonnier, Maldidier, Las Pélénos 2 et Brignol) où les grattoirs Caminade se présentent sans association avec d'autres marqueurs incontestables de la phase récente de l'Aurignacien'.

Malgré des études précédentes sur les grattoirs Caminade (Lucas 2000 ; Morala, Lenoir et Turq 2005 ; Caux 2015) et la chaîne opératoire de production de ceuxci (notamment Morala, Lenoir et Turq 2005 ; Caux 2015), les schémas opératoires présentés n'ont pas pu être documentés par des remontages. Ces derniers sont nécessaires pour compléter le schéma déjà proposé et pour explorer sa complémentarité ou cohérence relative vis-à-vis d'autres systèmes de production connus pour ce stade récent (Caux 2015). Brignol nous fournit ces remontages et nous permet donc de compléter nos connaissances sur ce schéma. En guise de conclusion, nous évoquerons quelques hypothèses au sujet du statut fonctionnel ou chronologique du faciès à grattoirs Caminade au sein de l'Aurignacien récent.

Une autre particularité de Brignol est qu'il s'agit d'un site de plein air. Or, les sites de plein air aurignaciens sont relativement peu exploités pour plusieurs raisons. La grande majorité de ces sites sont en réalité des collections de surface (Morala 1980, 1984 ; Bon et al. 2007) et ceux qui présentent une véritable stratigraphie ou une structuration spatiale sont rarement fouillés de manière exhaustive. Les quelques sites étudiés sont le plus souvent interprétés comme des ateliers de taille de complexité variable, tels que Champ-Parel 2 (Chadelle 1991, 2000, 2005) ou Corbiac-Vignoble 2 (Tixier et Reduron 1991 ; Tixier 1991) (Airvaux, Berthet et Castel 2003), comme des habitations ayant un fort effectif de production en début de chaîne laminaire, tels que Barbas (Ortega Cordellat et al. 2006) ou Chez Pinaud 2, comme des campements structurés de tailles variées, tels que Régismont-le-Haut (Bon 2002 ; Maurin 2006 ; Bon et al. 2007 ; Anderson et al. en prep.) ou, enfin, des campements sans aménagement conservé, tel quel Hui (Le Brun-Ricalens 2005). Brignol contribue donc aux réflexions sur le statut de ces sites de plein air durant l'Aurignacien : complémentarité fonctionnelle ou saisonnière avec les sites en abri ou expression de comportements semblables à plus haute résolution chronologique et spatiale?

Un troisième enjeu, étroitement lié au précédent, et qui sera à peine effleuré dans ce travail, est la possibilité d'extraire des informations d'ordre palethnologique du site de Brignol. Comme nous le verrons, le site est relativement bien conservé, et malgré quelques biais taphonomiques (cf. infra), une lecture spatiale relativement fine sera prochainement disponible.

(1) Mais de possibles déchets de taille liés à une production de type burin busqué ont été provisoirement identifiés au Pigeonnier, par A. Michel, et à Maldidier (Caux 2015). 


\section{2 - La fouille du site}

\section{1 - Contexte de l'intervention}

Préalablement à la construction du centre hospitalier de Villeneuve-sur-Lot, un diagnostic archéologique a été conduit par l'INRAP en mars 2011 sur un terrain d'environ 9 ha surmontant d'une quinzaine de mètres la rive droite du Lot (Ballarin et al. 2011) (fig. 1b). Une concentration d'objets lithiques a été découverte, alors attribuée prudemment au Gravettien ou au Magdalénien. Suite à une consultation, la société Paléotime a été retenue pour effectuer la fouille, sous la direction de R. Picavet, en novembre et décembre 2011. L'attribution culturelle de l'assemblage lithique à l'Aurignacien récent à grattoirs Caminade a alors été précisée par $\mathrm{O}$. Ferullo et $\mathrm{A}$. Morala.

L'environnement actuel du site est bordé au nord comme au sud de plateaux vallonnés. Ces reliefs relativement doux sont modelés dans des calcaires et des molasses tertiaires datées de l'Oligocène et du Miocène. Une petite falaise calcaire sépare le site de la rive du Lot. La prescription du Service régional de l'Archéologie d'Aquitaine portait sur une surface de $400 \mathrm{~m}^{2}$. La concentration se présente sous la forme d'un épandage de mobilier lithique, sans reste faunique conservé, situé à environ 1,60 $\mathrm{m}$ sous la surface actuelle. Aucune structuration évidente de l'espace n'a été identifiée lors de la fouille. Le contexte immédiat du site n'est pas complètement connu puisque d'autres concentrations de taille inférieure à la résolution des tranchées du diagnostic peuvent subsister sur le terrain (fig. 1b).

\section{2 - Méthode d'intervention}

La surface prescrite a été décapée mécaniquement jusqu'au toit des premiers vestiges puis une phase de reconnaissance de la concentration a été menée par la réalisation de 99 microsondages d'un quart de mètre carré sur la totalité de cette surface (fig. 2). Au total, la fouille s'est opérée manuellement sur $105 \mathrm{~m}^{2}$ (sondages compris). L'intégralité du sédiment excavé a été tamisée à l'eau sur une maille de $2 \mathrm{~mm}$ puis triée. Un décapage mécanique a été opéré à la fin de l'opération pour contrôle.

Une attention particulière a été portée sur la saisie topographique des objets, sans discrimination de taille, sur leur polarité ainsi que sur la mesure de fabriques des éléments allongés.

\section{3 - Contexte géoarchéologique et datation du site}

\section{1 - Séquence stratigraphique}

Le mobilier s'insère à la base d'une formation limonosableuse qui recouvre la terrasse Fw2 d'âge pléistocène moyen récent supposé (Capdeville et Turq 1999, fig. 1a). Les sept coupes stratigraphiques relevées ont permis de distinguer quatre principales unités pédosédimentaires qui se répartissent de manière homogène sur l'emprise de fouille. On trouve ainsi, de haut en bas (fig. 1c, 2 et 3a) :

- I'horizon cultivé actuel : limon sableux brun pâle (10YR6/3), épaisseur $35 \mathrm{~cm}$;

- un horizon éluvié, postérieurement brunifié par la pédogénèse actuelle : limon sableux brun jaune clair (10YR6/4) à brun (7.5YR5/4), sans éléments grossiers, d'épaisseur 20 à $40 \mathrm{~cm}$;

- un horizon argilique dégradé à la base duquel s'insère le mobilier : limon sablo-argileux brun sombre au sommet (7.5YR4/4) à brun vif à la base (7.5YR5/6), à graviers et petits galets siliceux dans la partie inférieure, d'épaisseur métrique ;

- le toit de la nappe alluviale Fw2 : graviers et galets siliceux (longueur maximale $10 \mathrm{~cm}$ ) pris dans une matrice sablolimoneuse brun vif (7.5YR5/6) (épaisseur observée $20 \mathrm{~cm}$ ).

Des fentes de dégradation s'ouvrent dans la partie supérieure de l'horizon argilique et affectent la nappe d'objets (fig. 1c). En plan, elles s'organisent selon un réseau polygonal inframétrique mal exprimé provoqué par la dessiccation du sol. Aucun trait cryogénique n'est associé à ce réseau. Les datations obtenues (cf. infra) et le niveau d'apparition de ces fentes impliquent une période de formation assez récente, dans tous les cas postérieure à la phase principale de développement du pergélisol attestée dans le Sud-Ouest (fin MIS3 à début MIS2 ; Bertran et al. 2013). En lame mince, le degré d'évolution de l'horizon argilique est cohérent avec une mise en place de cette séquence au cours du dernier cycle glaciaire.

\section{2 - Caractéristiques pédosédimentaires de l'horizon archéologique}

Aucune discontinuité stratigraphique ni variation significative de texture n'est perceptible au sein du volume contenant le mobilier lithique. De même, sur le terrain, aucune figure syn-sédimentaire n'a été décelée, aussi bien dans le dépôt limono-sableux que dans l'organisation des vestiges.

La fraction inférieure à $2 \mathrm{~mm}$ est composée par un limon $(64 \%)$ sablo- (24\%) argileux (12\%) mal trié, avec un mode principal situé autour de $14 \mu \mathrm{m}$. En lame mince, cette fraction est composée par un assemblage de particules quartzeuses subanguleuses, selon une microstructure de type porphyrique dense. Aucune figure de ruissellement, de déplacement en masse du sol ou de cryoturbation n'a été identifiée. L'homogénéité du matériau traduit vraisemblablement une aggradation progressive sous l'influence des processus de surface (bioturbation, gel/dégel, etc.). Différents agents ont pu contribuer à cette aggradation (crues, ruissellement, vent, bioturbation), sans que l'on puisse trancher entre ces différents mécanismes. Les traits illuviaux argileux, peu développés et en conformité avec la porosité, sont symptomatiques d'une longue évolution pédologique sous couverture forestière en contexte tempéré, évolution généralement rattachée à la première moitié de l'Holocène (Fedoroff et Courty 1987). Cette évolution explique le caractère acide du sol et en partie, la non-conservation des matériaux organiques. 


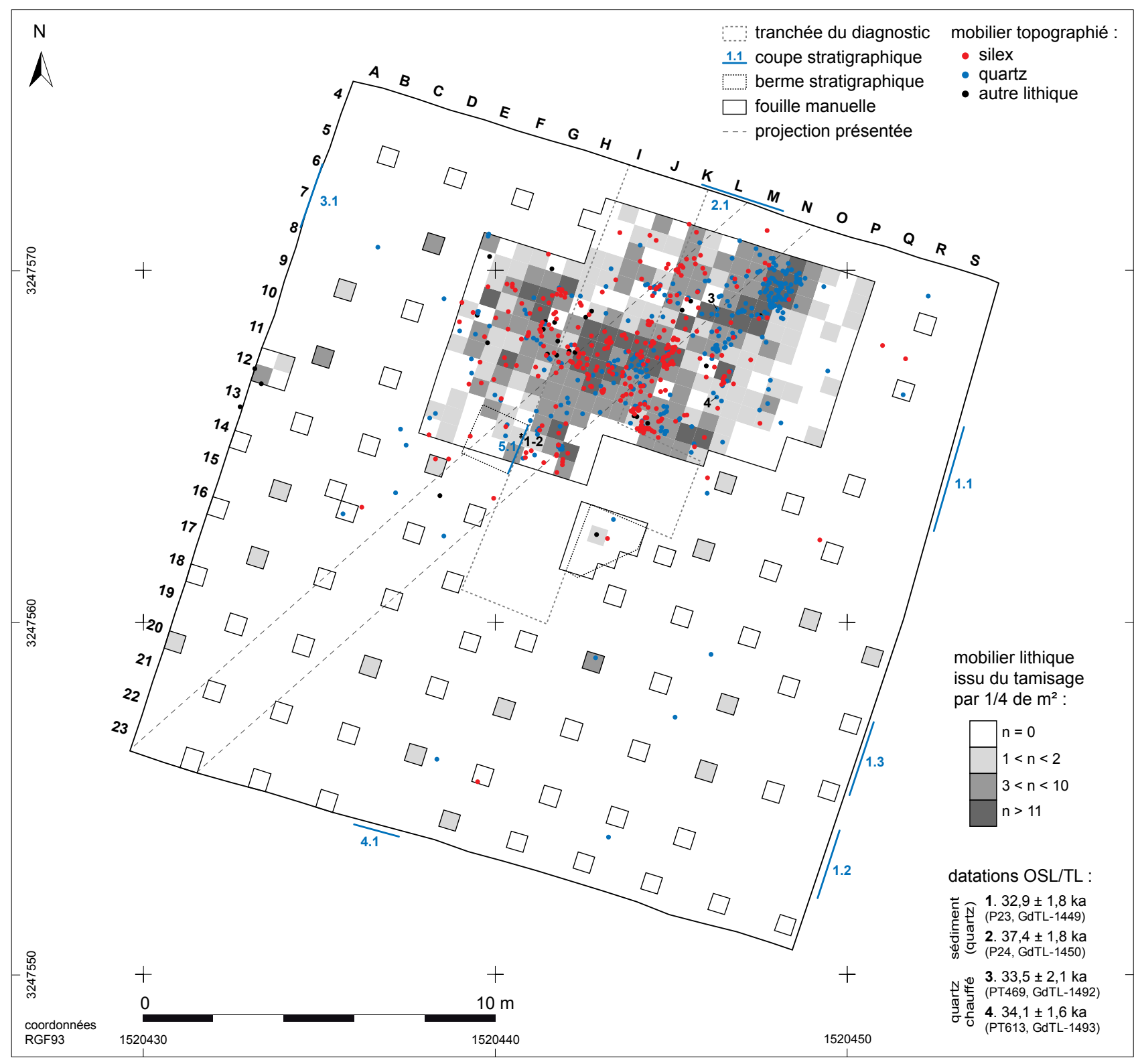

Figure 2 - Répartition en plan du mobilier lithique sur l'emprise de fouille (P. Tallet, J.-B. Caverne, M. Rué).

Figure 2 - Spatial repartition of lithic material across excavated surface (P. Tallet, J.-B. Caverne, M. Rué)

La fraction supérieure à $2 \mathrm{~mm}$, représentée principalement par des graviers et galets siliceux issus de la nappe alluviale, est de l'ordre de $1 \%$. Localement, cette fraction grossière se concentre en amas de taille décimétrique. Même si l'origine précise de ces concentrations nous échappe (remontée de matériaux par la bioturbation ? ruissellement concentré ? apports de crue ?), elles prouvent que des perturbations ont affecté la nappe lors des premiers stades de sa fossilisation. En plan, aucune corrélation n'existe toutefois entre la distribution de la fraction grossière et la répartition du mobilier.

\section{3 - Taphonomie lithique}

Le contexte pédosédimentaire, ainsi que l'absence de structuration évidente de l'espace lors de la fouille, ont motivé une étude visant à préciser les modalités de formation du site. Cette étude repose sur l'analyse spatiale du mobilier, la granulométrie de l'industrie lithique, l'étude des états de surface des silex et les remontages. L'analyse des fabriques n'était pas exploitable en raison d'un effectif de mesures trop faible. Les premiers et principaux résultats de cette étude sont exposés dans cette partie. 


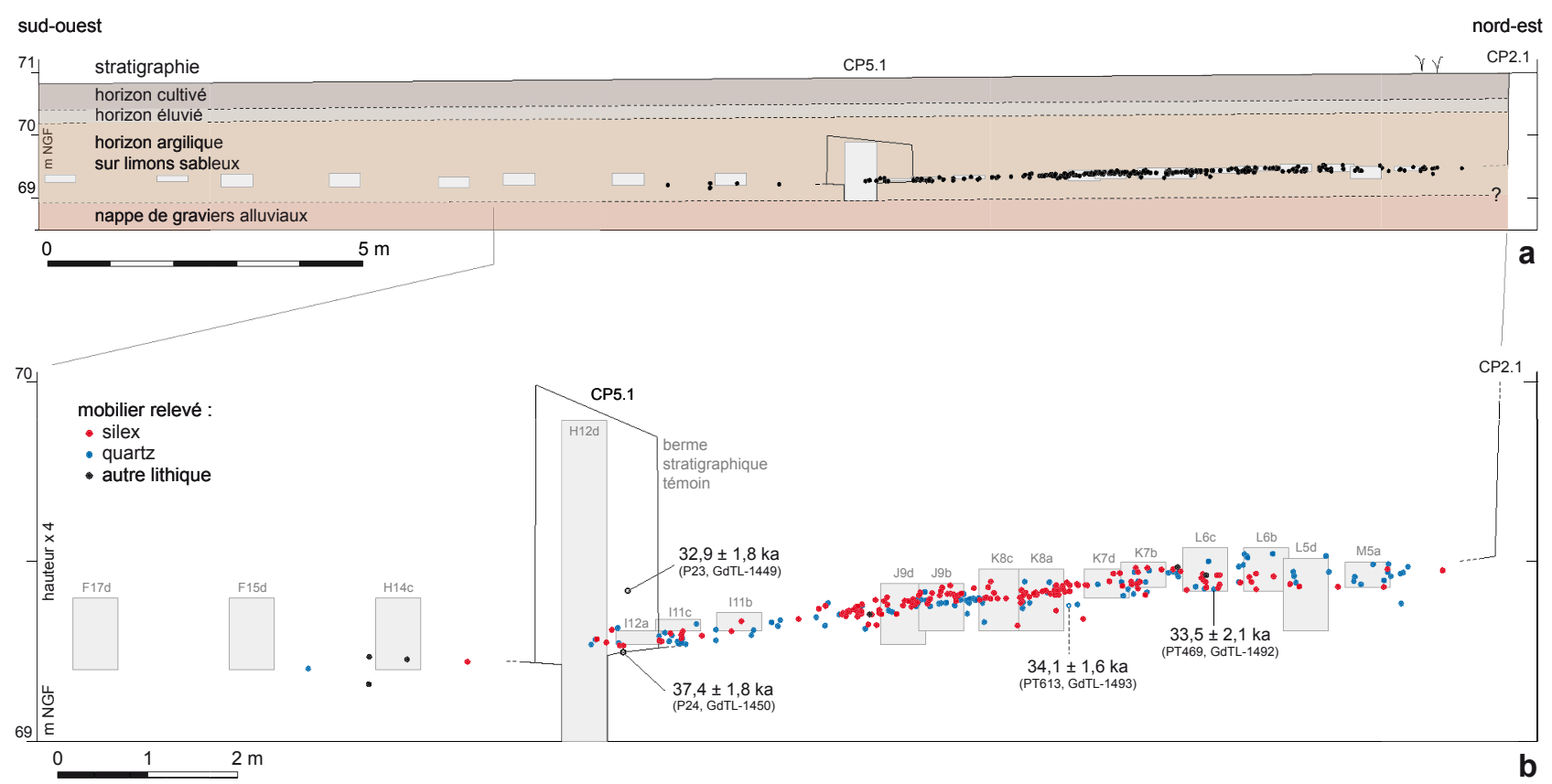

Figure 3 - Insertion de la nappe d'objets dans la séquence stratigraphique (M. Rué, J.-B. Caverne). Projection verticale du mobilier présent sur une largeur de $2 \mathrm{~m}$ et d'une sélection de sous-carrés fouillés. Axe de projection localisé dans le sens de la plus grande pente de la nappe (localisation dans fig. 2). a. Ensemble de l'emprise. b. Détail au niveau de la concentration et localisation des dates OSL/TL. Hauteur multipliée par 4.

Figure 3 - Position of archaeological level within stratigraphic sequence (M. Rué, J.-B. Caverne). Vertical projection of archaeological materials from a $2 \mathrm{~m}$ wide cross-section of excavated sub-squares. Projection axis localized in the direction of the strongest slope of archaeological materials (localisation in fig. 2). a. Profile of entire surface tested. b. Detail of archaeological level and localisation of OSL/TL dates. Height multiplied by 4.

Précisons au préalable que la fouille manuelle n'a pas complètement circonscrit la concentration lithique. L'épandage de mobilier se poursuit probablement vers le nord-est. Une partie du mobilier a également disparu lors du creusement de la tranchée de diagnostic et de son extension orientale (fig. 2). Le mobilier n'a donc pas été recueilli exhaustivement, dans des proportions difficiles à estimer, mais sans doute faibles au regard du plan de distribution des objets. On discerne en effet relativement bien le pourtour d'une unique concentration couvrant une surface d'environ $80 \mathrm{~m}^{2}$ (fig. 2). D'après l'étude lithique, différents regroupements d'objets ont par ailleurs été identifiés en plan. Cette organisation spatiale prouve donc que les processus naturels n'ont pas complètement homogénéisé l'assemblage initial.

L'épandage de mobilier forme un plan incliné d'environ $2^{\circ}$ en direction du sud-ouest, dans le sens de la pente naturelle et est situé à environ $30 \mathrm{~cm}$ au-dessus de la nappe alluviale. Un léger replat est perceptible dans la partie nord de la fenêtre de fouille manuelle. La dilatation verticale du mobilier relevé au théodolite est globalement homogène sur la surface fouillée, elle n'excède généralement pas $12 \mathrm{~cm}$. Certains éléments ont toutefois pu migrer le long des fentes ou d'anciens conduits biologiques. Pour preuve, un petit éclat de silex provient du fond du sondage $\mathrm{H} 12 \mathrm{~d}$, soit une vingtaine de centimètres sous la concentration (fig. 3b).

En plan, aucun tri granulométrique évident n'apparaît. La répartition de la fraction issue du tamisage coïncide relativement bien avec celle de la fraction topographiée (fig. 2). La forme de la concentration est toutefois légèrement allongée dans le sens de la plus grande pente, ce qui traduit de probables migrations d'objets et explique le plus grand nombre de pièces isolées dans la zone aval.

L'analyse granulométrique du mobilier, réalisée selon le protocole proposé par Bertran et al. (2012), est basée sur le mobilier issu du tamisage de 66 quarts de mètre carré répartis sur l'ensemble de la concentration. La distribution obtenue montre un déficit de la fraction 2-4 mm et renvoie l'échantillon dans la zone des premiers stades de résidualisation, en dehors de l'aire des débitages expérimentaux, qu'il s'agisse des éléments en silex ou en quartz (fig. 4). Ce déficit peut toutefois s'expliquer par la chaine opératoire et la spécificité des activités menées sur le site dont on sait qu'elles peuvent influencer significativement le signal granulométrique (jusqu'à $35 \%$ d'après Todisco, Bhiry et Desrosiers 2009).

L'analyse des états de surfaces des pièces a été conduite sur un échantillon représentatif de la série lithique lors de son étude $(n>200)$. La recherche de stigmates d'altération 


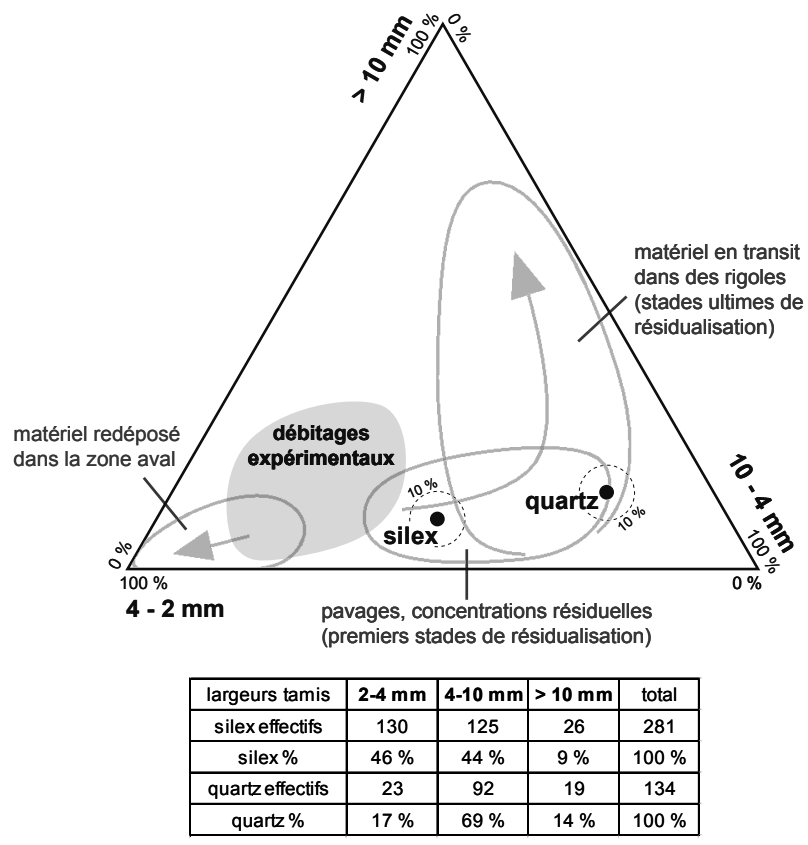

Figure 4 - Distribution granulométrique du mobilier ( $P$. Tallet, $M$. Rué). Diagramme ternaire des classes dimensionnelles d'après Bertran et al. 2012.

Figure 4 - Granulometric distribution of archaeological materials (P. Tallet, M. Rué). Ternary distribution of dimensional classes according to Bertran et al. 2012.

mécanique sur les bords et les nervures des pièces a donné des résultats disparates. $35 \%$ des pièces montrent des bords altérés alors que les stigmates sont très faibles sur les nervures. II faut également noter l'absence de stigmates liés à l'alternance gel/dégel, observation qu'il faut pondérer en raison de la taille réduite des pièces. Le type de silex mis en œuvre est pourtant sensible à la cryoclastie (Coutard 2002). Une patine blanche touche un quart du mobilier et un lustré d'origine chimique s'est développé sur la totalité des pièces, sans polarisation. Les altérations mécaniques et physico-chimiques, décelables sur les bords et les nervures/surfaces des objets, empêchent une lecture microscopique des traces, mais comme l'on verra (cf. infra), une lecture des macro-traces est toutefois possible. L'ensemble de ces indicateurs suggère que la phase d'enfouissement ait pu s'opérer dans un délai relativement court après l'abandon des pièces, et probablement en contexte tempéré (Vallin et al. 2013).

Un investissement important a été consacré à la recherche de remontages et raccords. Les taux de remontage obtenus sont relativement élevés, respectivement de $32,7 \%$ des silex remontables, $(19,2 \%$ de l'intégralité de l'ensemble des silex recueillis) et $22 \%$ pour les quartz remontables. La distribution des axes de remontages met en évidence une orientation préférentielle significative lorsque l'on prend en compte la totalité des silex $(p<0,05)$ (fig. 5). Cette orientation coïncide avec celle de la pente de la nappe de mobilier, ce qui conforte l'hypothèse d'une redistribution des objets par

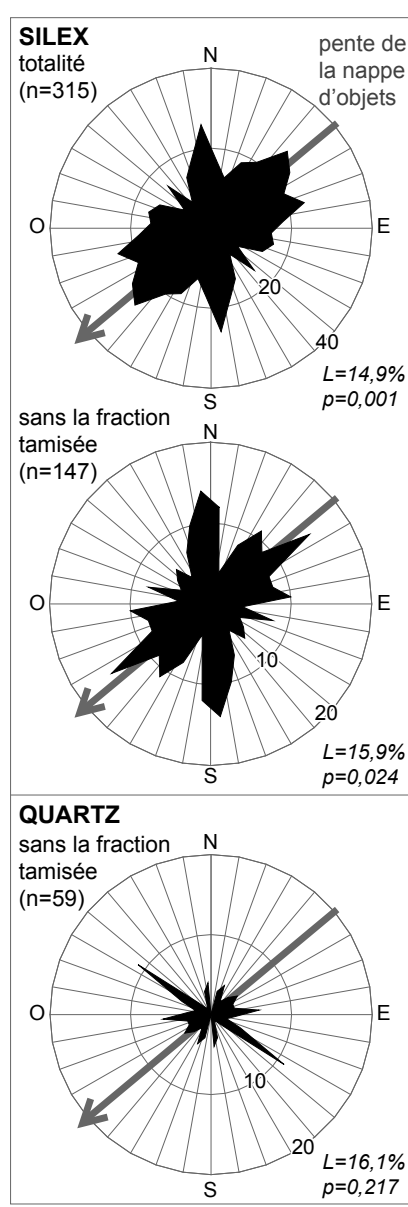

Figure 5 - Distribution de l'orientation des axes de remontages (L. Anderson, E. Kawalec, M. Rué). $L$ : intensité de l'orientation préférentielle (vector magnitude) d'après Curray (1956) ; $p$ : probabilité pour que la distribution des orientations soit le seul fait du hasard.

Figure 5 - Distribution of refit axis orientations

(L. Anderson, E. Kawalec,

M. Rué). L: intensity of preferential orientation (vector magnitude) according to Curray (1956); $p$ : probability that preferential orientations are random.

les processus de surface. Le fait que les éléments en quartz ne soient pas ou peu impactés par la pente peut s'expliquer en partie par leur morphologie non aplatie, les rendant moins sensibles au transport.

\section{4 - Datations par luminescence}

Deux volumes de sédiment et deux quartz chauffés ont fait l'objet d'une datation OSL/TL par l'Institut de Physique de Gliwice (Pologne) (localisation des prélèvements fig. 2 et 3 ). Les doses équivalentes ont été déterminées selon le protocole single-aliquot regenerative-dose (Murray et Wintle 2000). Les taux d'irradiation ont été estimés en laboratoire, sans dosimétrie in situ. Les échantillons proviennent toutefois d'un environnement pédosédimentaire homogène. L'estimation des variations de la teneur en eau du sédiment au cours du temps est par ailleurs très difficile à estimer dans ce contexte alluvial. Pour ces raisons, les écarts-types fournis par le laboratoire nous apparaissent sous-estimés. D'après les résultats, le site semble être intégralement recouvert par les limons à partir de $33 \mathrm{ka} \mathrm{BP}$ et la période de dernière chauffe des quartz se situe autour de 34 ka BP (fig. 6).

Même si ces résultats sont à prendre avec précaution en raison des incertitudes liées aux mesures des doses de radiation, les deux dates obtenues sur les quartz chauffés 


\begin{tabular}{|c|c|c|c|c|c|c|c|c|c|c|c|c|c|c|c|c|}
\hline \multirow[b]{2}{*}{$\mathrm{n}^{\circ}$} & \multirow[b]{2}{*}{$\begin{array}{l}\text { référence } \\
\text { laboratoire }\end{array}$} & \multirow[b]{2}{*}{ matériau } & \multirow[b]{2}{*}{$\begin{array}{l}\text { altitude } \\
\text { (m NGF) }\end{array}$} & \multirow[b]{2}{*}{$\begin{array}{c}\text { prof. I } \\
\text { surface } \\
\text { actuelle } \\
\text { (m) }\end{array}$} & \multirow[b]{2}{*}{$\begin{array}{c}\text { teneur en } \\
\text { eau } \\
\text { moyenne } \\
\text { estimée } \\
(\%)\end{array}$} & \multirow[b]{2}{*}{$\begin{array}{c}\text { nom bre } \\
\text { d'aliquotes }\end{array}$} & \multicolumn{3}{|c|}{$\begin{array}{l}\text { Activité du sédiment environnant } \\
\qquad(\mathrm{Bq} / \mathrm{kg})\end{array}$} & \multicolumn{5}{|c|}{ Débit de dose (Gy/ka) } & \multirow[b]{2}{*}{$\begin{array}{c}\text { paléodose } \\
\text { (Gy) }\end{array}$} & \multirow[b]{2}{*}{$\begin{array}{l}\text { âge (ka) } \\
\text { BP }\end{array}$} \\
\hline & & & & & & & Th-232 & $\mathrm{U}-238$ & $K-40$ & alpha & beta & gamma & cosmique & total & & \\
\hline P23 & GdTL-1449 & quartz $125-200 \mu \mathrm{m}$ & 69,42 & 1,45 & $18 \pm 4$ & 14 & $44,0 \pm 1,1$ & $34,67 \pm 0,62$ & $642 \pm 18$ & $0,065 \pm 0,01$ & $1,743 \pm 0,09$ & $1,120 \pm 0,03$ & $0,179 \pm 0,02$ & $3,106 \pm 0,09$ & $102,4 \pm 4,4$ & $32,9 \pm 1,8$ \\
\hline P24 & GdTL-1450 & quartz $125-200 \mu \mathrm{m}$ & 69,25 & 1,62 & $18 \pm 4$ & 17 & $42,95 \pm 0,85$ & $32,86 \pm 0,58$ & $657 \pm 18$ & $0,062 \pm 0,01$ & $1,754 \pm 0,09$ & $1,106 \pm 0,03$ & $0,174 \pm 0,02$ & $3,097 \pm 0,09$ & $116,1 \pm 4,2$ & $37,4 \pm 1,8$ \\
\hline PT469 & GdTL-1492 & quartz chauffé & 69,42 & 1,61 & - & 12 & $47,16 \pm 0,84$ & $33,96 \pm 0,56$ & $664 \pm 18$ & 0 & 0 & $1,255 \pm 0,04$ & $0,174 \pm 0,02$ & $1,429 \pm 0,04$ & $48,0 \pm 2,6$ & $33,5 \pm 2,1$ \\
\hline PT613 & GdTL-1493 & quartz chauffé & 69,38 & 1,61 & - & 13 & $50,4 \pm 1,1$ & $35,02 \pm 0,62$ & $742 \pm 21$ & 0 & 0 & $1,354 \pm 0,05$ & $0,174 \pm 0,02$ & $1,528 \pm 0,05$ & $52,1 \pm 1,8$ & $34,1 \pm 1,6$ \\
\hline
\end{tabular}

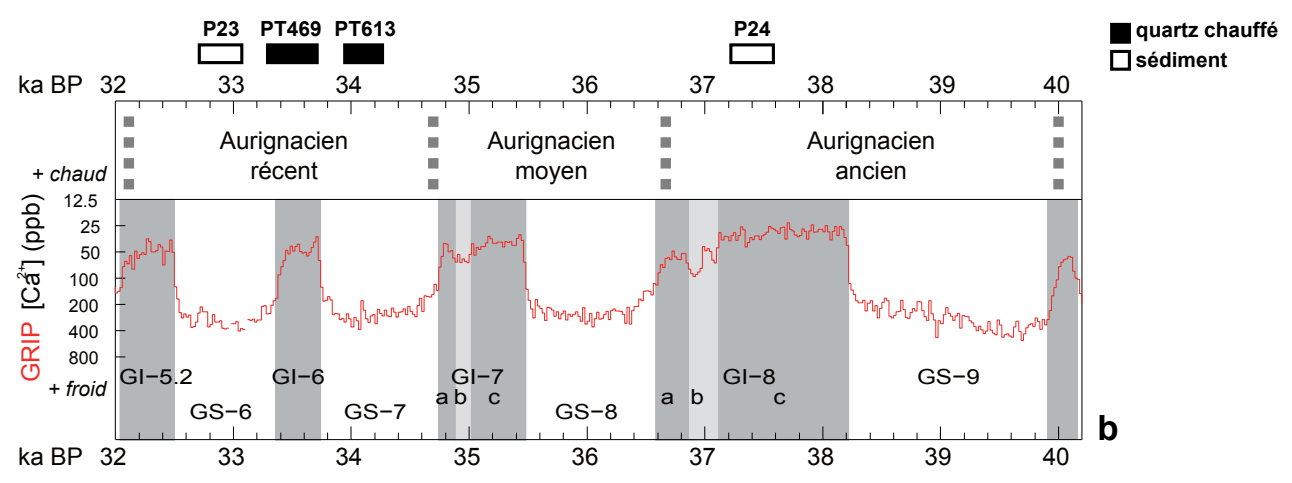

Figure 6 - Datation du site par luminescence. a. Tableau des résultats (G. Adamiec, Gliwice Absolute Dating Methods Centre). b. Répartition des dates. Stades (GS), interstades (GI) et courbe d'après Rasmussen et al. 2014, chronologie de l'Aurignacien d'après Michel 2010.

Figure 6 - Site dating by luminescence. a. Table of results (G. Adamiec, Gliwice Absolute Dating Methods Centre). b. Localisation of dates. Stadials (GS), interstadials (GI), and curve according to Rasmussen et al. 2014, Aurignacian chronological synthesis according to Michel 2010.

sont cohérentes avec une attribution de l'industrie à l'Aurignacien récent, ceci en considérant les associations stratigraphiques qui existent pour ce technocomplexe dans d'autres sites. Les datations absolues sont encore rares pour ce faciès de l'Aurignacien. Elles seront à confirmer par d'autres travaux.

\section{5 - Conclusion}

À ce stade de l'analyse, l'approche géoarchéologique menée sur le site de Brignol permet d'avancer les résultats suivants :

1. Le mobilier s'insère à la base d'un luvisol dégradé qui s'est développé sur des apports limoneux d'origines variées datés par OSL de la fin du stade 3 , en accord avec l'âge de l'industrie. L'homogénéité de ces dépôts ne fournit pas d'indicateur évident sur le milieu de dépôt initial, et donc sur le contexte paléoenvironnemental, même si l'on sait qu'il se situe en zone inondable, et que le site est donc épisodiquement inaccessible. Les états de surface des silex plaident en faveur d'un recouvrement précoce du site, probablement en contexte tempéré (absence de stigmate de gel/dégel et de réorganisation périglaciaire des vestiges).

2. La distribution spatiale et les taux de remontage montrent que l'assemblage initial n'a pas été complètement modifié par les processus géomorphologiques. Néanmoins, d'après la distribution granulométrique et l'orientation des remontages, une partie du mobilier a subi des déplacements dans le sens de la pente, sans doute limités dans l'espace. Certaines catégories d'objets ont probablement migré en dehors de la concentration.

3. La relative bonne homogénéité des états de surface, le taux élevé de remontages, le caractère circonscrit et réduit de la concentration principale ainsi que la cohérence technologique et typologique (cf. infra) montrent que l'on est en présence d'un ensemble taphonomique homogène, ce qui autorise une lecture fine de l'assemblage recueilli.

\section{4 - Étude lithique}

\section{1 - Présentation générale}

La série de Brignol consiste en 2061 objets lithiques, dont 1242 en silex (731 éléments étudiables et 511 débris et esquilles indéterminés inférieurs à $1 \mathrm{~cm}$ issus des refus de tamis) et 781 en quartz (objets côtés et issus des refus de tamis). À ce chiffre se rajoutent quelques éléments en d'autres matières premières, telles que le quartzite ou le calcaire gréseux. La production en silex s'oriente principalement vers l'obtention d'éclats lamellaire, mais une petite production laminaire (fig. 7), ainsi qu'un petit effectif d'outils du fonds commun domestique, sont également présents.

Ces éclats lamellaires sont parfois transformés en grattoirs Caminade, une catégorie qui domine le profil typologique 


\begin{tabular}{|c|c|c|c|c|c|c|c|c|c|c|c|c|}
\hline Catégorie technologique (vertical) par matière première (horizontal) & ষ্ণ & 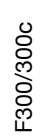 & 啇 & 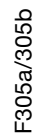 & 命 & 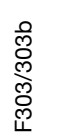 & 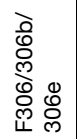 & : & $\stackrel{6}{\frac{0}{m}}$ & ণ్రి & 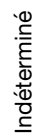 & ॠू \\
\hline $\begin{array}{l}\text { Blocs/fragments de bloc } \\
\text {...dont nucléus à grattoirs Caminade } \\
\text {...dont nucléus à grattoirs Caminade simplifiés } \\
\text {...dont nucléus à lames } \\
\text {...dont nucléus lamino-lamellaires simplifiés } \\
\text {...dont blocs testés } \\
\text {...dont nucléus préformés/testés/avortés }\end{array}$ & & & & & & $\begin{array}{l}2 \\
2\end{array}$ & $\begin{array}{l}5 \\
1 \\
2 \\
1\end{array}$ & & & $\begin{array}{l}6 \\
1 \\
2 \\
4\end{array}$ & & $\begin{array}{l}13 \\
2 \\
1 \\
1 \\
4 \\
1 \\
4\end{array}$ \\
\hline $\begin{array}{l}\text { Éclats } \\
\ldots \text {...ont allongés } \\
\text {...dont nucléus préformés/testés/avortés }\end{array}$ & 4 & 7 & 6 & 1 & & 46 & $\begin{array}{l}37 \\
3\end{array}$ & 1 & 6 & $\begin{array}{l}108 \\
2 \\
1\end{array}$ & 1 & $\begin{array}{l}217 \\
5 \\
1\end{array}$ \\
\hline $\begin{array}{l}\text { Éclats épais... } \\
\text {...dont nucléus à grattoirs Caminade } \\
\text {...dont nucléus à grattoirs Caminade sur éclat roulé avec double patine } \\
\text {...dont nucléus lamino-lamellaires simplifiés } \\
\text {...dont nucléus préformés/testés/avortés }\end{array}$ & $\begin{array}{l}1 \\
1\end{array}$ & & $\begin{array}{l}2 \\
1\end{array}$ & & & $\begin{array}{l}1 \\
1\end{array}$ & & & $\begin{array}{l}1 \\
1\end{array}$ & $\begin{array}{l}6 \\
2 \\
1 \\
2\end{array}$ & & $\begin{array}{l}11 \\
5 \\
1 \\
1 \\
2\end{array}$ \\
\hline Lamelles & 5 & 8 & & 1 & & 15 & 7 & & 6 & 29 & 2 & 73 \\
\hline Éclats lamellaires & 17 & 25 & 3 & 4 & 5 & 57 & 10 & 5 & 25 & 66 & 3 & 220 \\
\hline $\begin{array}{l}\text { Lames... } \\
\text {...dont nucléus à grattoirs Caminade sur lame épaisse }\end{array}$ & & $\begin{array}{l}2 \\
1\end{array}$ & 1 & 2 & & & & & & $\begin{array}{l}22 \\
1\end{array}$ & 3 & $\begin{array}{l}30 \\
2\end{array}$ \\
\hline Éclats laminaires & & & & & & & 4 & & & 6 & & 10 \\
\hline Éclats/lamelles de retouche probable & & 4 & & & & 2 & 1 & & & 96 & 1 & 104 \\
\hline Indéterminés & 1 & & & & & 1 & 5 & & & 40 & 6 & 53 \\
\hline Total & 28 & 46 & 12 & 8 & 5 & 124 & 69 & 6 & 38 & 379 & 16 & 731 \\
\hline
\end{tabular}

Note : Étude pétroarchéologique menée par $\mathrm{P}$. Fernandes sur 591 pièces ; étude technologique de l'ensemble et attribution des matières premières pour 140 pièces restantes (surtout par critères macroscopiques) menées par $\mathrm{L}$. Anderson (le décompte final des matières premières est donc approximatif). Codes de matières premières selon Fernandes et al. (2013) : F304 (Campanien 1-2, groupe des silex de Belvès) ; F300/300c (Campanien 4-5, groupe des silex du Bergeracois) ; F301 (groupe des silex du Campanien supérieur ; F305a/305b (Turonien, groupe des silex du Fumélois) ; F307 (groupe des silex jaspéroïdes) ; F303/303b (groupe des silex lacustres) ; F306/306b/306e/309 (groupe des silex du Santonien) ; F316 (groupe des silex type Séguine, altérites du Santonien) ; F302 (silex Cénozoïques, groupe des calcédoines)

Figure 7 - Décomptes de l'industrie en silex par catégorie technologique et matière première (L. Anderson).

Figure 7 - Counts of flint lithic industry by technological category and raw material (L. Anderson).

de l'ensemble (cf. infra), auquel s'ajoutent six grattoirs sur lame/éclat laminaire et cinq lames retouchées, ainsi qu'une pièce esquillée. L'outillage sur éclat est également très peu représenté, avec un grattoir sur éclat gelifracté et cinq éclats retouchés. Les différents types de grattoirs épais/aurignaciens $(n=10)$, tous classés comme des nucléus suite aux remontages et à l'étude technologique (fig. 7), sont représentés majoritairement par des variantes circulaires ou sub-circulaires $(n=5)$, suivis numériquement par le type " grattoir caréné » $(n=3)$ et par les grattoirs double $(n=1)$ ou simple $(n=1)$ sur support épais. Aucun produit en quartz ne fut retouché. Le cas est semblable pour les quartzites et les calcaires gréseux, avec un seul macro-outil dans ce dernier.

\section{2 - Les quartz taillés et chauffés}

À Brignol, les Aurignaciens ont collecté des galets de quartz dans les flancs d'érosion de la proche terrasse du Lot. L'absence totale de ce genre de galets en dehors de l'emprise archéologique fouillée nous démontre que la totalité de ces objets a été amenée sur le site par les préhistoriques. Nous avons donc choisi de traiter le quartz comme le résultat d'un choix pétrographique et technique destiné à des fonctions particulières par les Aurignaciens. Au stade de la fouille, nous pouvions classer les quartz en deux familles distinctes : les galets fracturés par la chauffe et les galets taillés. Ce constat nous a amenés à repérer spatialement chaque débris de quartz, toutes catégories confondues, à des fins techniques et d'étude spatiale.

Concernant les quartz chauffés, nous avons opté pour l'appellation "culinaire», d'après Bosinski et Guicharnaud (2008), partant de l'idée que ces galets chauffés auraient pu être utilisés pour chauffer de l'eau et cuire des aliments, les excluant de la série des galets taillés.

Nous nous sommes inspirés des travaux de D. Batchelor et de ses observations réalisées dans le cadre de l'étude des quartz du site de Gönnersdorf (Batchelor 1979) et basées sur une série d'expérimentations. Nous avons, à notre tour, réalisé une expérimentation de chauffe et de taille des galets de quartz dont le protocole a été adapté en utilisant des matériaux de la même provenance que ceux utilisés par les Aurignaciens de Brignol. D'après l'observation qualitative, les stigmates de chauffe expérimentaux et archéologiques semblent identiques (à savoir une fragmentation polyédrique, une rubéfaction et des haircracks généralisés), ce qui tend à valider l'hypothèse d'une utilisation par chauffe progressive dans un foyer suivi par une immersion dans l'eau (fig. 8).

L'intégralité de la chaîne opératoire de la taille des quartz est présente sur les lieux. Le débitage est orienté dans le sens 

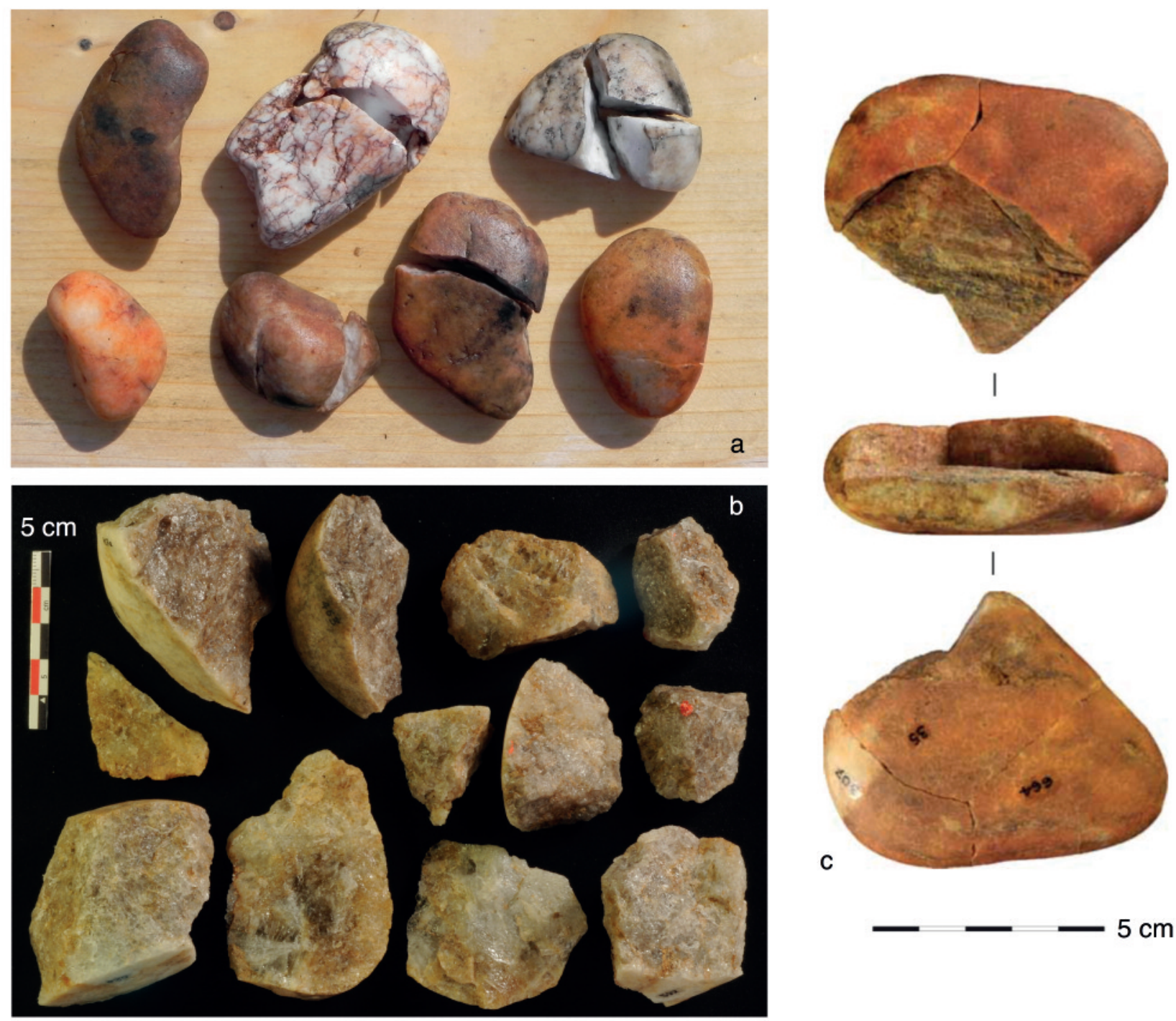

C

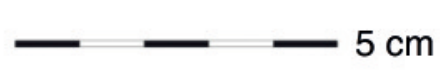

Figure 8- Quartz chauffés ; exemplaires expérimentaux (a) et archéologiques (b et c) (Photographies : R. Picavet).

Figure 8 - Heated quartz; expérimental examples (a) and archaeological examples (b and c) (Photographs: R. Picavet).

des microdiaclases (souvent orientées transversalement par rapport à la longueur du galet), et l'entame se fait en extrémité du galet choisi. Le débitage d'éclats, à la percussion directe dure, progresse ainsi de manière alternée tant que la préhension du nucléus est toujours possible.

\section{3 - Les autres matériaux lithiques, hors silex}

Les quartzites, également présents dans les alluvions éocènes de la terrasse du Lot, ont été peu récoltés, de même que les galets de granite. Parmi les blocs non travaillés, trois galets de taille importante de calcaire gréseux dont deux ont été découverts en place lors de la fouille manuelle, disposés à plat sur l'horizon aurignacien, sont issus de la terrasse et complètent cette série. Quatre autres blocs de calcaire gréseux ont été travaillés. Pour deux d'entre eux, il s'agit de nucléus macrolithiques à éclats exploités selon les mêmes modalités que les galets de quartz, et un troisième est débité de manière anarchique sans véritable production d'éclats, mais de polyèdres épais. Le quatrième bloc, un « chopper » de forme naturellement triangulaire, présente un fort émoussé et un esquillement sur la face opposée de son arête retouchée. II s'agit d'un macro-outil, fabriqué en matériau fragile ayant probablement été utilisé par percussions sur enclume.

\section{4 - Étude pétroarchéologique des objets en silex}

L'étude pétroarchéologique des objets en silex de Brignol a permis d'esquisser les limites du domaine minéral exploité par ses occupants. La méthodologie pétroarchéologique appliquée consiste en l'étude de la " chaîne évolutive » des silicifications utilisées par les groupes préhistoriques (e.g. Fernandes 2012). 
L'examen de 591 pièces archéologiques (suite à l'étude technologique) sur un total de 1242 comparées à 29 échantillons géologiques régionaux a permis de sérier 14 types. Les types gitologiques fixés sont regroupés en 14 groupes $^{2}$ ayant chacun une origine géographique différente (reposant sur les référentiels lithologiques (Morala 1980, 1984 ; étude P. Fernandes in Picavet et al. 2013). Ce travail a permis de déterminer la provenance géologique de la majorité des objets lithiques étudiés (574 sur 591).

Les occupants de Brignol ont collecté principalement des silcrètes issues des calcaires de l'Agenais connues sous les noms de meulières ou calcédoine $\left(\mathrm{F} 302^{3}\right)$. Les formations qui contiennent ce type de silicifications se trouvent, à proximité, notamment en rive droite du Lot. L'origine exacte du deuxième groupe d'objets (F303 et F303b) n'a pas été déterminée. II s'agit de silex lacustres que nous attribuons à un ensemble comprenant les formations de Montbazillac, de Castillon et du bassin de la Lède. Le reste du corpus confirme la fréquentation des alluvions du Lot, de la Thèze et de la Lémance (F305, F305b, F306, F306e, F306b, F309b), ceux de la Dordogne et de la Couze (F301, F300c, F300), mais aussi le secteur autour de Belvès ou de la vallée du Dropt (F304) et possiblement de la forêt de Monclard, ou encore la vallée de la Lède vers Blanquefort-sur-Briolance (F316). Nous n'avons cependant retrouvé aucun de ces types dans l'espace strictement local.

Les indices lithologiques et l'aspect des états de surface regroupés au cours de cette étude livrent un schéma territorial qui correspond à un transect sud-nord entre la vallée du Lot et la vallée de la Dordogne. Le site se trouve, a priori, aux limites méridionales d'un domaine minéral exploité sur 50 kilomètres, au sein duquel certains affluents du Lot et de la Dordogne ont joué un rôle important. Les occupants de Brignol possédaient donc une bonne connaissance de la variabilité régionale, mais ont tout de même fait le choix de privilégier un matériau local, représenté dans une sphère géographique d'une vingtaine de kilomètres de rayon.

\section{5 - La gestion des matières premières siliceuses}

Si l'application du terme « économie des matières premières " (Perlès 1991) s'avère délicate pendant l'Aurignacien, on observe toutefois une gestion des matières premières très spécifique au cours de cette période (Anderson et al. 2015). Couplée avec des sousstades de divergences et convergences entre les chaînes opératoires laminaires et lamellaires, respectivement attribuées aux sphères domestique et cynégétique (Tartar et al. 2006), cette gestion différentielle des matières premières renvoie aux différentes stratégies de mobilités employées par les Aurignaciens (Anderson et al. 2015 ; Bordes, Bon et Le Brun-Ricalens 2005).

Dès l'Aurignacien ancien, une forte dissociation lames/lamelles se met en place, ce qui le distingue assez nettement du Protoaurignacien qui le précède. La production laminaire s'oriente vers l'obtention de produits robustes, typiquement destinés à la confection de l'outillage domestique, tandis que la production lamellaire semble se tourner vers la confection d'armatures modulaires (Bon 2005) pour des armes de jet (O'Farrell 2005 ; Normand, O'Farrell et Rios Garaizar et al. 2008), a priori utilisées dans la sphère cynégétique. En ce qui concerne la gestion des matières premières, malgré des productions laminaires qui peuvent être effectuées sur place, surtout dans les régions riches en roches siliceuses de grande qualité, les matières premières allochtones sont normalement représentées par des outils laminaires déjà confectionnés, parfois très loin de leurs origines gîtologiques (jusqu'à $300-400 \mathrm{~km}$ à vol d'oiseau) (Féblot-Augustins 2008). Le cas est inversé pour les productions lamellaires. Majoritairement en silex locaux et régionaux, il apparaît que les lamelles sont produites au gré des besoins avec les matières disponibles, alors qu'on observe une forte anticipation des futurs besoins dans l'industrie laminaire (Bon 2005). Cette gestion différentielle des sphères domestiques et cynégétiques est plus prononcée en dehors de la région classique du nord du Bassin aquitain. Les sites de la Tuto de Camalhot (Vézian et Vézian 1966 ; Bon 2002 ; Bon, Simonnet et Vézian 2005 ; Bordes, Bon, Le Brun-Ricalens 2005) et de Régismont-le-Haut (Bordes, Bon et Le Brun-Ricalens 2005 ; Féblot-Augustins 2008) sont de très bons exemples. Cette forte anticipation des besoins domestiques, opposée à une certaine flexibilité dans l'industrie lamellaire, renvoie probablement à un cycle annuel de nomadisme caractérisé par une mobilité résidentielle très structurée, sans doute assez planifiée (Anderson et al. 2015). Cette dichotomie lames/lamelles et la gestion particulière des matières premières semblent continuer pendant les phases récentes de l'Aurignacien (Michel 2010), mais de manière moins radicale, surtout concernant le faciès à burins busqués (Caux 2015).

Bien qu'une dissociation entre l'industrie laminaire et lamellaire soit présente à Brignol, la gestion des matières premières ne renvoie pas aux comportements mentionnés ci-dessus. Tout d'abord, il n'y a pas de véritable silex extrarégional dans la série. Les silex locaux sont privilégiés, et ceux qui ne sont pas disponibles dans un rayon de $20 \mathrm{~km}$ autour du site le sont entre 25 et $50 \mathrm{~km}$ à vol d'oiseau, tels que le Fumélois (F305, F305b) et le Bergeracois du Campanien supérieur (F300, F300c) (Morala 1980, 1984 ; étude P. Fernandes in Picavet et al. 2013).

(2) Un type gîtologique est défini par ses transformations prédépositionelles, alors qu'un groupe correspond à une association d'objets possédant au moins un trait commun rapportable à un domaine géologique.

(3) Les codes utilisés font référence à l'atlas des types traceurs en Aquitaine établi dans Fernandes et al. (2013). 
Le débitage laminaire, très peu représenté sur les lieux, est mené majoritairement avec les calcédoines locales (F302) disponibles en grande quantité en rive droite du Lot. Seulement quatre outils ou fragments d'outil sur lame (environ $7 \%$ de l'ensemble laminaire) sont présents en silex extralocaux, dont deux en Turonien du Fumélois (F305, F305b). Les productions lamino-lamellaires simplifiées (cf. infra), toutes effectuées en calcédoines (F302) ou santoniens locaux (F306, F306b, F306e, F309b) témoignent d'une activité exclusivement locale. Enfin, c'est la production lamellaire, représentée par la chaîne opératoire des grattoirs Caminade à Brignol, qui présente surtout une forte composante extralocale.

Parmi les séquences lamellaires attribuables à une production de grattoirs Caminade, quatre des dix nucléus probables (dont huit remontés entièrement ou en partie) sont issus des formations primaires ou des alluvions situées entre 25 et $50 \mathrm{~km}$ au nord du site (F301, F304, F300c, F316). Si l'on rajoute une petite séquence de production de grattoirs Caminade en Fumélois (F305, F305b) représentée par quelques déchets techniques et un produit retouché (ce qui implique l'emport du nucléus en dehors du site ou dans une zone non fouillée), la proportion de silex extralocal employée dans l'industrie lamellaire s'élève à environ $31 \%$ (de l'ensemble dans son intégralité, mais $40 \%$ des nucléus lamellaires). Trois nucléus en silex lacustre, disponible dans les formations entre le Dropt et le Lot (dans un rayon de 0 à $25 \mathrm{~km}$ du site) (F303, F303b), représentent également environ $30 \%$ de l'ensemble lamellaire. Parmi les silex locaux, seulement trois nucléus, dont deux sans remontage, et une forte proportion de déchets techniques en calcédoine, représentent environ $31 \%$ de l'ensemble lamellaire. Enfin, cinq grattoirs Caminade en silex d'aspect jaspéroïde (F307) abandonnés sur les lieux, sans déchet technique ni nucléus, témoignent de leur débitage et emmanchement en amont de l'occupation. Malheureusement, l'origine précise de ces pièces reste inconnue ; des matériaux de ce type se trouvent dans les basses terrasses du Lot (Fernandes in Picavet et al. 2013) mais sont également connus dans des formations de l'Infralias de la marge occidentale du Massif central (Demars 1980 ; Turq 1999, 2000).

II est intéressant de noter qu'aucune matière première importée en quantité sur les lieux ne provient des régions situées au sud du Lot. Or, les silex venant de plus loin sont plus fortement représentés par des nucléus abandonnés et leurs déchets techniques, alors qu'il y a relativement peu de nucléus par rapport à la quantité de déchets lamellaires dans les silex strictement locaux. Cela souligne une arrivée du groupe vraisemblablement depuis le nord, avec des grattoirs Caminade déjà emmanchés. Cela témoigne également d'une anticipation des besoins concernant la production de grattoirs Caminade pour leur remplacement en cours d'utilisation, et d'un possible réapprovisionnement en silex local.

Cette gestion s'éloigne de la norme décrite pour l'Aurignacien (cf. supra). Une anticipation des besoins lamellaires ainsi qu'une production d'outillage domestique minime (sur lame et sur éclat à Brignol) renvoient à la prévision d'une tâche spécifique concernant les grattoirs Caminade et un rôle secondaire pour le fonds commun domestique. Ce schéma n'est toutefois pas la norme pour les sites à grattoirs Caminade non plus. Ceux de la vallée de la Dordogne témoignent d'une gestion standard pour l'Aurignacien récent, alors que ceux en dehors de cette même vallée (Brignol ou Le Pigeonnier par exemple) témoignent de cette anticipation lamellaire (Caux 2015). Est-ce à mettre en lien avec le statut particulier de ces sites ? II est donc nécessaire de s'interroger sur le statut fonctionnel de ces objets particuliers avant de s'interroger sur le statut du site en lui-même. Une supposition que ces objets appartiennent à la sphère cynégétique, comme c'est souvent le cas pour les industries lamellaires de l'Aurignacien, influencera sans doute notre interprétation du site. Cependant, cette association n'est pas encore démontrée.

\section{6 - Les productions laminaires}

\subsection{1 - Chaîne laminaire et son intersection avec l'outillage domestique}

L'unique nucléus à lames et la présence d'éclats corticaux et de quelques déchets techniques dans un même faciès de calcédoine locale démontrent que ce type de production est présent, mais qu'il est toutefois d'intérêt secondaire sur les lieux.

Le débitage du nucléus est unipolaire et frontal avec une table plate et relativement cintrée, ainsi que des flancs corticaux (fig. 9). Le plan de frappe, légèrement facetté et entretenu par l'enlèvement des tablettes, forme un angle assez fermé par rapport à la table de débitage, ce qui entraînerait un carénage distal relativement accentué des lames robustes détachées. Pour les lames de plein débitage, souvent à pan cortical, les talons sont fréquemment facettés, voire en éperon, plus rarement lisses, et les corniches sont abrasées préalablement à leur détachement à l'aide d'un percuteur tendre. Les gestes d'entretien, par contre, semblent être effectués sans préparation à l'aide d'un percuteur dur. La réorientation et la reprise du nucléus par un tailleur peu expérimenté empêchent la lecture des possibles crêtes postérieures ou néocrêtes antérieures qui auraient pu servir respectivement à cintrer ou à réaménager la table laminaire, mais une évaluation morphotechnique des produits laminaires (Bon 2002) de la série nous montre que des crêtes médianes d'entame, ainsi que des néocrêtes antérolatérales en cours de débitage, sont présentes, mais peu fréquentes. Plus courant pour l'entretien du cintre est le recours aux lames débordantes, souvent à pan cortical. Malgré un effectif laminaire réduit, cette modalité d'entretien nous semble être le plus souvent latéralisée à gauche, ce qui est confirmé par un débordement plus fort des enlèvements laminaires sur le flanc gauche du seul véritable nucléus laminaire.

L'outillage sur lame à proprement parler est proportionnellement peu représenté par rapport aux 


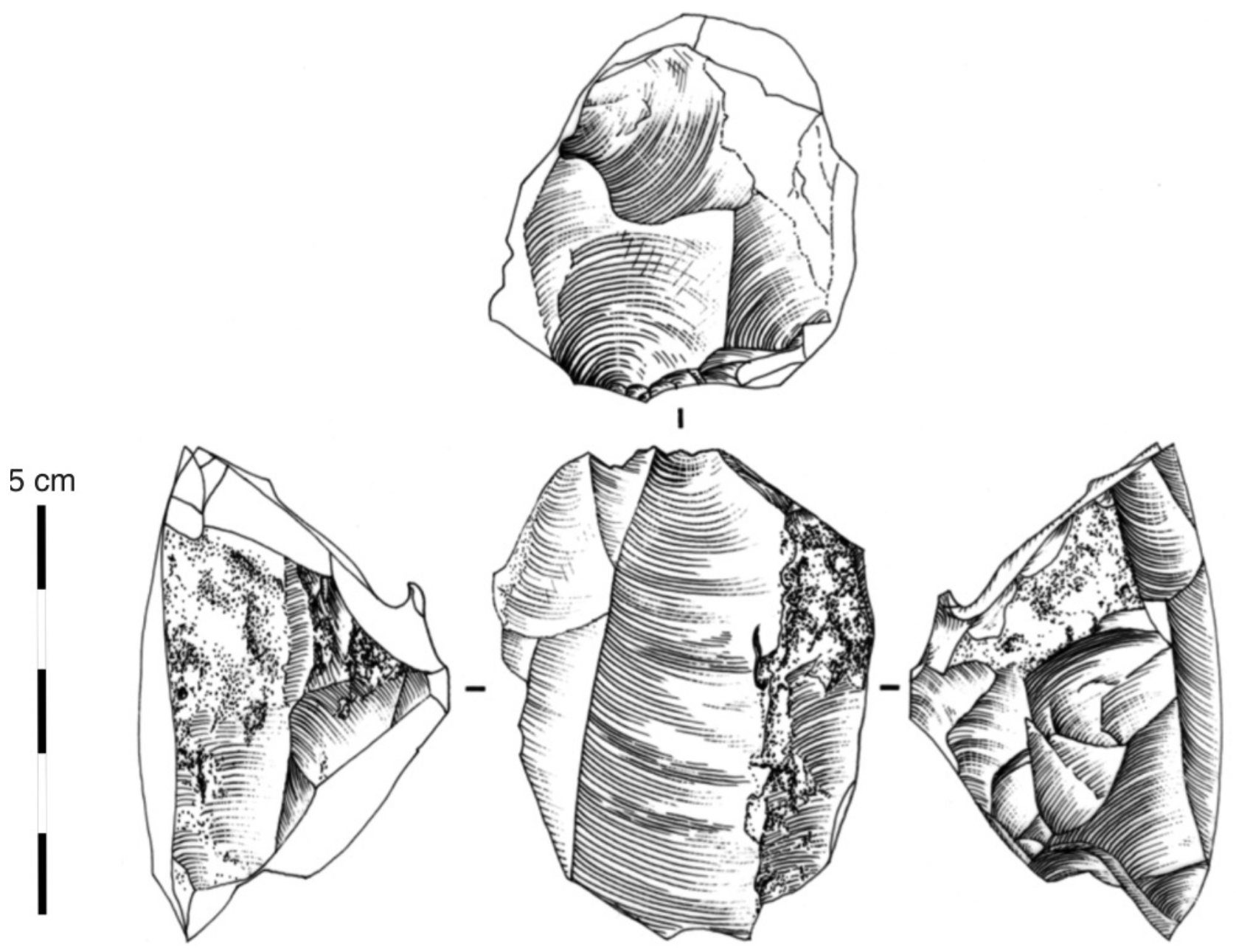

Figure 9 - Unique nucléus à lames de l'ensemble lithique de Brignol (Dessin : R. Picavet).

Figure 9 - Unique blade core from the Brignol lithic assemblage (Drawing: R. Picavet).

produits retouchés issus de la chaîne lamellaire, et une bonne partie semble être débitée en amont de l'occupation ou dans une zone non fouillée. Ce petit effectif consiste en quelques grattoirs sur lame ou sur éclat laminaire (entiers ou fragmentés, $n=6$ ), quelques produits laminaires retouchés $(n=5$, dont un portant de véritables retouches aurignaciennes, et un autre étant vraisemblablement la partie proximale d'un grattoir sur lame), et finalement un grattoir en silex du Bergeracois repris en pièce esquillée. Un petit effectif d'éclats retouchés ( $\mathrm{n}=6$, dont un véritable grattoir sur éclat gélifracté), sur des supports au moins en partie sélectionnés dans les déchets techniques issus de la chaîne laminaire, vient compléter l'outillage domestique plus traditionnel.

\subsection{2 - Débitages lamino-lamellaires simplifiés}

Parallèlement à l'effectif réduit de pièces témoignant d'un vrai débitage laminaire, le débitage lamino-lamellaire évoque selon nous un véritable manque de savoir-faire (sensu Pelegrin 1991). Quatre séquences remontées quasi intégralement (et un nucléus avec un seul remontage) témoignent de ce débitage qui, à nos yeux, ne semble pas avoir d'authentiques objectifs économiques. Dans ces quatre exemples, les matrices initiales sont appréhendées de la même manière ; quel que soit le support initial, une face étroite est choisie comme table de débitage (fig. 10). Un enlèvement « burinant » permet l'installation de l'unique plan de frappe perpendiculaire à la future table de débitage (l'ancienne table est transformée en plan de frappe pour le nucléus laminaire repris). Cette dernière est entamée à l'aide d'une crête médiane à un ou deux versants, ou tout simplement par le détachement d'un éclat laminaire entièrement cortical, les deux stratégies privilégiant une percussion dure. La poursuite du débitage, intégralement à la pierre dure, démontre un manque de précision et de contrôle du geste (qui se manifeste parfois par quelques cônes incipients sur le plan de frappe qui semblent précéder le détachement du produit souhaité), ce qui entraîne des produits irréguliers/réfléchis et une perte 
a

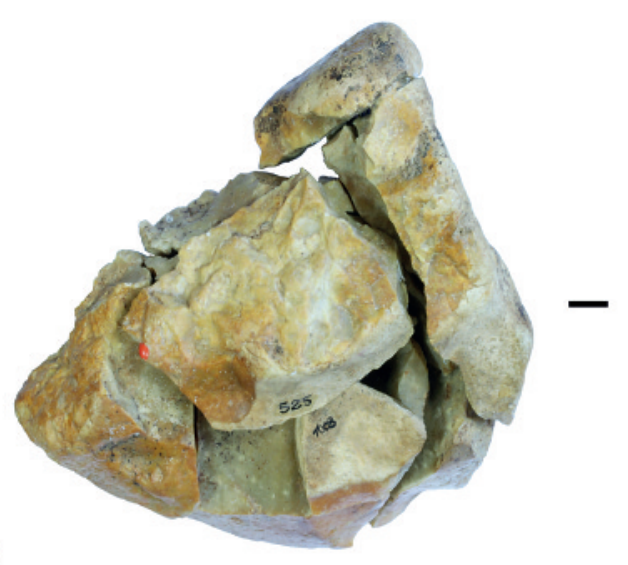

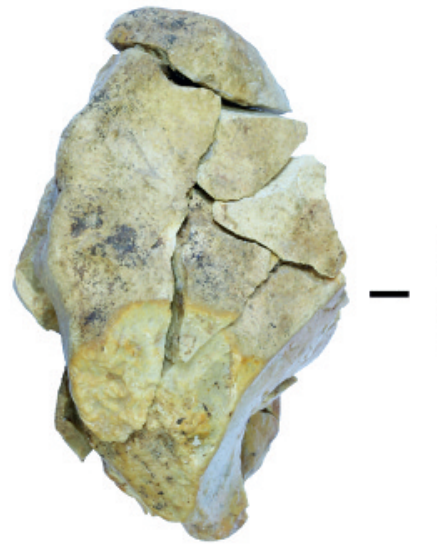

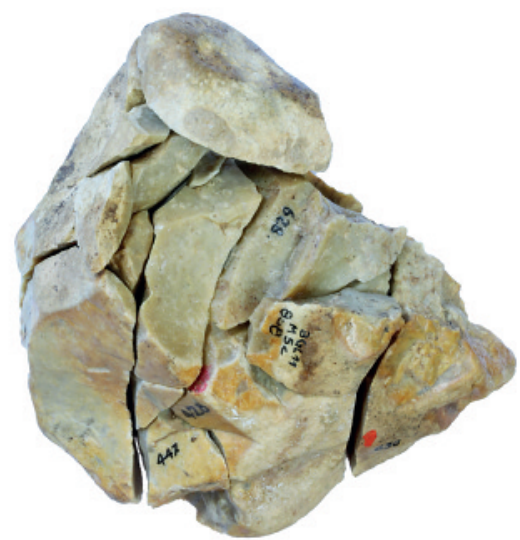

$5 \mathrm{~cm}$

b
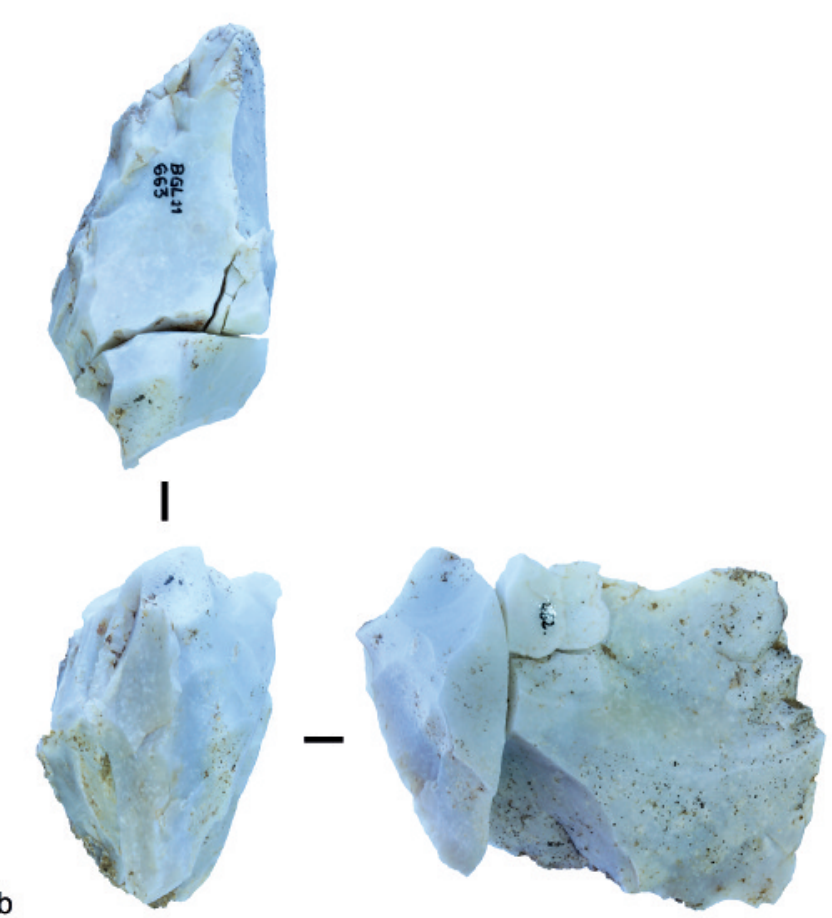

Figure 10 - Deux exemples de remontages lamino-lamellaires simplifiés/mal exécutés, dont un (a) sur bloc alluvial de santonien et un deuxième (b) sur tranche d'éclat épais en calcédoine (L. Anderson).

Figure 10 - Two examples of simplified/poorly executed blade/bladelet refits, the first (a) a rolled alluvial block of santonian flint and the second (b) a thick flake of chalcedony exploited in a burin-like fashion (L. Anderson).

relativement rapide des propriétés volumétriques idoines. Sans acharnement particulier, le nucléus est soit abandonné prématurément, soit réorienté pour une nouvelle séquence de débitage. Un des blocs, en silex santonien de basse qualité (fig. 10a), a trois stades de vie, montrant tous les mêmes types de maladresses. Cela pourrait témoigner du même auteur pour les trois phases. Or, un autre bloc dans ce même faciès de santonien de basse qualité, ayant la même morphologie initiale, est seulement testé. Ceci évoque deux différents degrés de savoir-faire, quelqu'un qui peut voir que la qualité de la matière empêchera un débitage réussi, et un autre qui n'a pas encore intégré cette notion, ou n'a tout simplement pas accès aux blocs de meilleure qualité sur les lieux, vu leur rareté. 

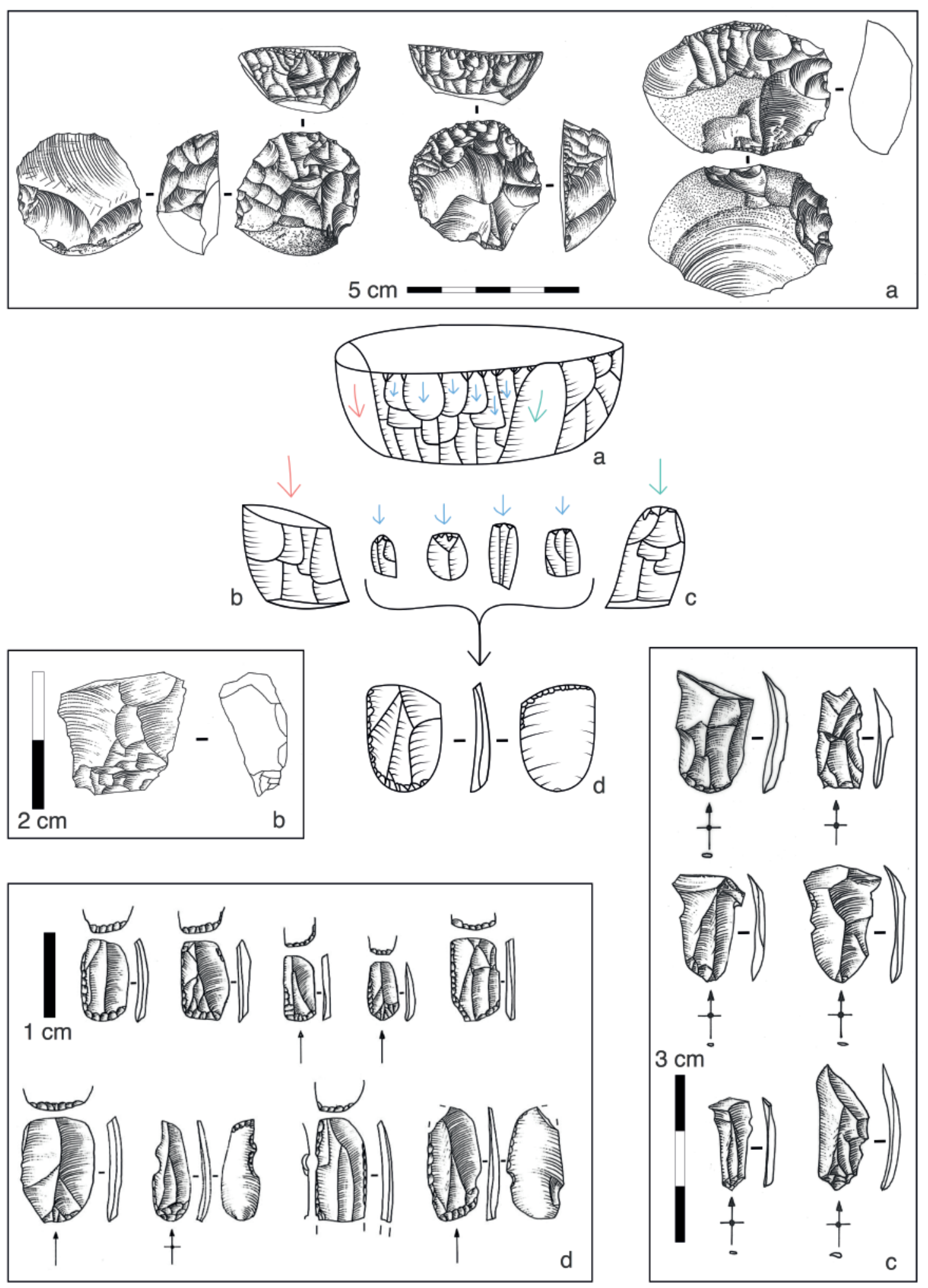

Figure 11 - Schéma opératoire synthétique de production de grattoirs Caminade : a. exemples de nucléus à grattoirs Caminade ; b. encoche d'entretien ; c. éclats lamellaires de ménage ; d. produits sélectionnés et transformés en grattoirs Caminade (Schéma opératoire : L. Anderson; Dessins ; R. Picavet).

Figure 11 - Synthesized operational sequence of grattoirs Caminade production: a. examples of grattoir Caminade cores; b. maintenance notch; c. bladelet-like maintenance flakes ; products selected and transformed into grattoirs Caminade (Operational sequence: L. Anderson ; Dessins: R. Picavet). 


\section{7 - Le débitage lamellaire}

\subsection{1 - Le schéma opératoire des nucléus à grattoirs Caminade (fig. 11)}

La production de supports destinés à être transformés en grattoirs Caminade a déjà fait l'objet d'études technologiques (notamment par Lenoir et Turq (Morala, Lenoir et Turq 2005 et Caux 2015). La chaîne opératoire de production des grattoirs Caminade à partir de grattoirs-nucléus ou racloirsnucléus ${ }^{4}$ a été reconstituée pour la première fois sur le site du Pigeonnier (Gensac, Gironde) par Morala, Lenoir et Turq (2005).

Cependant, l'absence de remontages témoignant de cette production n'a pas permis une description complète de cette chaîne microlithique de l'Aurignacien récent. La série de Brignol est réduite, mais le taux élevé de remontages en roches siliceuses (32,7\% des silex remontables ou 19,2\% de l'intégralité de l'ensemble des silex), à savoir dix nucléus à supports de grattoirs Caminade probables, dont huit entièrement ou en partie remontés, nous permet de compléter nos connaissances sur ce schéma opératoire.

Comme c'est souvent le cas pour la relation entre chaînes laminaires et lamellaires pour les industries postprotoaurignaciennes, derrière le terme « indépendant » se cache un certain degré de variabilité. Les schémas opératoires laminaires et lamellaires à Brignol sont bel et bien indépendants, mais la sélection des supports-nucléus lamellaires les rapproche ponctuellement. Celle-ci démontre en effet une récupération des sous-produits et déchets de taille laminaires pour un réemploi en tant que nucléus lamellaire (conjointement à une production indépendante de supports-nucléus sur petit bloc). Un certain degré d'opportunisme est donc décelable dans cette sélection.

Les supports privilégiés pour les nucléus à grattoirs Caminade à Brignol sont majoritairement des éclats épais ou blocs/fragments de blocs subcirculaires. Dans le premier cas, la face inférieure de l'éclat est utilisée comme plan de frappe, et dans le second cas, le négatif d'un enlèvement ou une surface d'éclatement du bloc initial peuvent également servir comme plan de frappe. La récupération de lames épaisses et possiblement de déchets de taille d'une chaîne laminaire effectuée ailleurs démontre également l'aspect opportuniste de la sélection des supports. Malgré la variabilité des supports initiaux, il apparaît que la mise en place de la surface de débitage et les gestes d'entretien qui suivent privilégient une table de débitage courte et trapézoïdale, généralement entre 1,4 et $1,9 \mathrm{~cm}$ de long. La recherche d'une table courte est à mettre en lien avec le produit recherché, ainsi que les modalités d'entretien mises en œuvre pour maximiser la productivité. Les nucléus ayant des tables plus longues, ceux de morphologie « grattoir caréné », démontrent tout de même, au moins dans leurs dernières générations de production, un même objectif.

La mise en forme de cette table courte et trapézoïdale semble être relativement sommaire. Parmi les huit nucléus partiellement ou entièrement remontés, seulement trois possèdent les phases initiales d'entame. Dans le premier cas, sur éclat épais, quelques éclats réfléchis, détachés depuis la face inférieure de l'éclat, créent une aspérité transverse dans la partie distale de la future table. Pour enlever cette aspérité transverse, le tailleur détache une encoche légèrement outrepassée en frappant un peu en recul du bord du plan de frappe. La mise en place de cette aspérité transverse, couplée avec un geste rentrant, induisent l'outrepassement contrôlé de l'encoche, mais aussi l'étalement latéral de l'onde de choc en partie distale (fig. 12). Cette encoche, de morphologie trapézoïdale, permet donc de mettre en place la morphologie souhaitée de la table et d'obtenir une bonne angulation entre la table lamellaire et le plan de frappe qui est normalement proche de $60^{\circ}$. Un deuxième exemple de cette stratégie d'entame montre le détachement d'un premier éclat d'entame qui a les mêmes propriétés que celles mentionnées ci-dessus, mais suivant une mise en forme différente.

Dans ce deuxième exemple, le tailleur aménage une crête distale transverse à un versant qui joue le même rôle que l'aspérité transverse décrite ci-dessus. Même si l'intention initiale du tailleur n'est peut-être pas un débitage de supports pour grattoirs Caminade, il exploite cette crête distale transverse de la même manière que l'aspérité transverse déjà décrite (fig. 12). Le fait que le plan de frappe semble être aménagé avant l'enlèvement de cet éclat d'entame et que la crête en elle-même n'est jamais exploitée en tant que crête médiane/antérolatérale plaident en faveur d'une véritable opération d'entame pour une production de type grattoirs Caminade. Suite à l'enlèvement de cet éclat d'entame, une néocrête antérolatérale à un versant est aménagée sur la future table, et une suite d'éclats outrepassés, qui emportent avec eux également une partie de la crête distale transverse, servent à mettre en place une table lamellaire trapézoïdale avec une bonne convexité transverse (fig. 12). Malgré l'aspect anecdotique de ces deux exemples, ils confirment l'intention initiale d'obtenir une table trapézoïdale ayant un bord de plan de frappe qui forme un arc de cercle, ce qui peut être fait également avec de simples encoches outrepassées si la morphologie du support-nucléus est adaptée. Ceci est le cas pour le troisième exemple où un bloc aplati est entamé par simple encoche corticale.

Quoi qu'il en soit, une fois entamés, ces nucléus produisent des supports de grattoirs Caminade à l'aide d'une abrasion soignée du bord du plan de frappe et, vraisemblablement, d'un percuteur tendre organique assez dense. Malgré une reprise relativement fréquente du talon $(20 \%$ des 54 produits étudiés), ce qui empêche sa lecture, le 


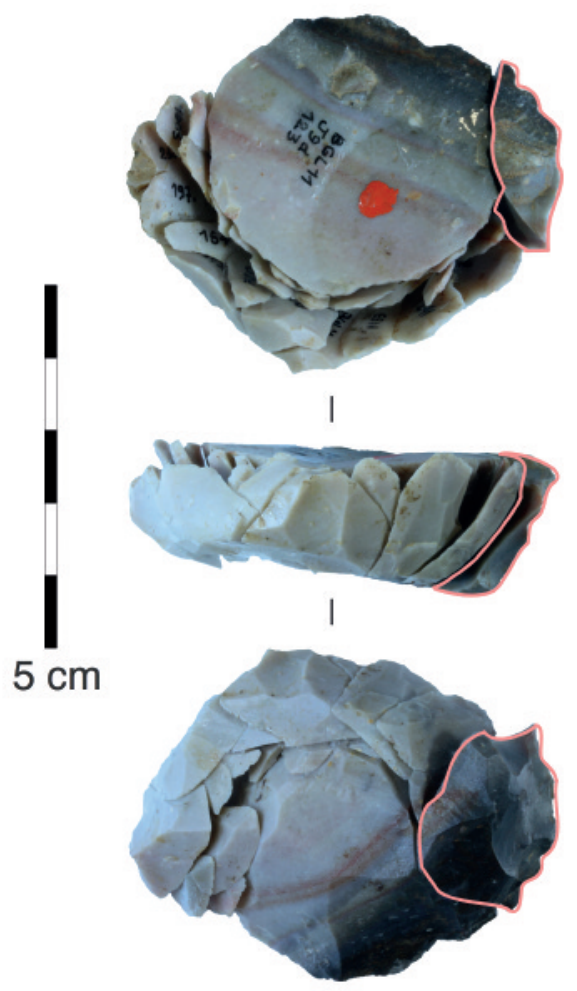

$1 \mathrm{a}$

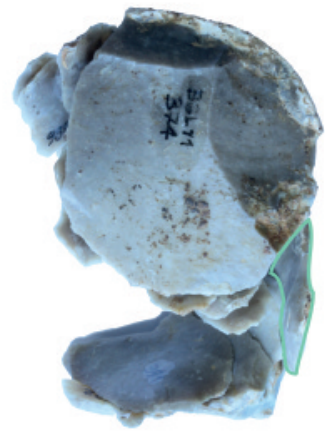

I

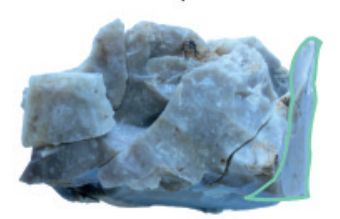

।

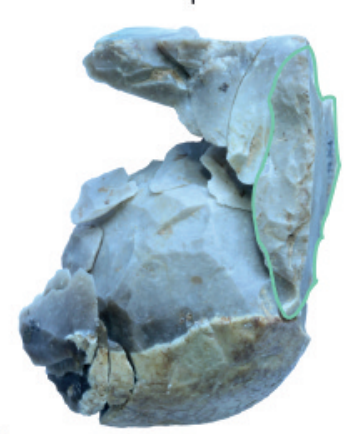

$2 \mathrm{a}$
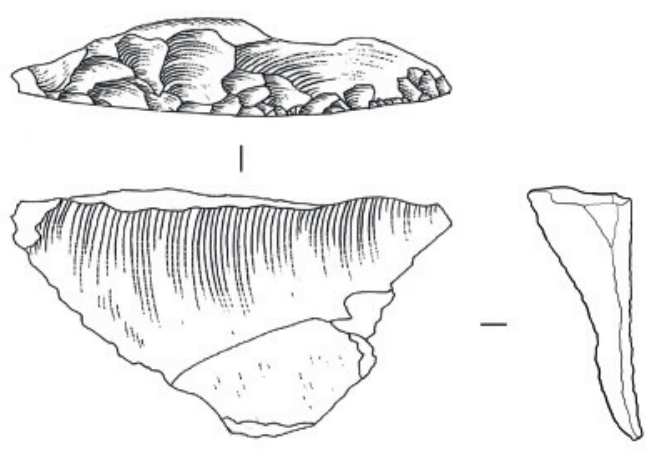

$2 \mathrm{~b}$

Figure 12 - Remontages sur nucléus à grattoirs Caminade ; éléments détourés en photographies 1a et 2a sont dessinés (1b et $2 b)$; 1b. encoche légèrement outrepassée avec aspérité transverse distale en premières phases de production ; $2 b$. éclat d'entame outrepassée de table lamellaire avec crête distale transverse (Photographies : L. Anderson ; Dessins : R. Picavet).

Figure 12 - Refits on grattoirs Caminade cores; elements outlined in photographs 1a and 2a are drawn (1b and 2b); 1b. Lightly plunging notch with distal transverse ridge during first phases of production; $2 b$. plunging opening flake of bladelet surface with distal transverse crest (Photographs : L. Anderson; Drawings : R. Picavet) 
relativement grand nombre de très petits talons lisses $(29 \%)$, voire punctiformes (14,8\%) abrasés, ayant parfois des petites lèvres soutiennent cette hypothèse de technique de détachement. Quelques produits recherchés issus d'un geste légèrement plus rentrant soutiennent également cette hypothèse, avec des lèvres plus marquées et des talons lisses abrasés relativement larges témoignant de l'étalement du point de contact caractéristique d'une utilisation de percuteur tendre organique (Pelegrin 2000).

L'étude des remontages couplée avec l'étude de la chronologie des enlèvements sur la face supérieure des produits recherchés (qui ne montre au passage aucun schéma de latéralisation préférentielle des gestes en amont du détachement de ceux-ci) nous indiquent qu'une fois initialisées, les générations de production lamellaire semblent suivre une progression de $1 / 4$ à 1/3 tournant symétrique (Valentin, Weber et Bodu 2014). La poursuite du débitage de cette manière entraîne un aplatissement de la table, alors qu'un produit recherché souvent légèrement réfléchi induit une accumulation de petits réfléchissements sur la table et une perte graduelle de l'angle et de la carène. Pour surmonter cela et permettre la conduite du débitage, des éclats lamellaires de nettoyage sont détachés à l'aide d'un percuteur tendre organique et d'un geste un peu plus rentrant. Ces déchets techniques sont assez caractéristiques (fig. 11). Un geste plus rentrant associé à une légère aspérité sur la face supérieure en partie mésiodistal, le résultat de l'accumulation de petits réfléchissements, entraînent un léger outrepassement contrôlé et une onde de choc qui s'étale latéralement en partie distale. En conséquence, ces éclats lamellaires de nettoyage sont souvent légèrement outrepassés. Ils ont également une série de petits réfléchissements sur leurs faces supérieures en partie mésiodistale et peuvent être plus larges en partie distale (fig. 11c). Ce geste d'entretien sert dans un premier temps à corriger les pertes de carène, mais peut aussi avoir un rôle secondaire concernant l'entretien du cintre. Néanmoins, quand ces éclats lamellaires de nettoyage ne sont pas suffisants pour l'entretien du cintre, un geste encore plus rentrant permet de détacher de véritables encoches, souvent avec les mêmes propriétés que les premiers, mais aux dépens d'une plus forte épaisseur (fig. 11b). Ces encoches peuvent également ponctuer différentes générations de production lamellaire, puisqu'elles servent à dégager un front ayant une bonne convexité transverse (tout comme les gestes de mise en forme de la table décrits ci-dessus).

Cette manière de procéder, combinée avec une sélection de supports de nucléus relativement circulaires, entraînent la migration latérale de la table lamellaire au cours de la vie du nucléus le plus souvent vers la droite, selon les remontages et les schémas diacritiques. Cela implique la succession de débitage de séries de lamelles avec une progression de $1 / 4$ à $1 / 3$ tournant symétrique, mais une progression plus générale de $1 / 2$ jusqu'à entièrement tournant asymétrique au cours de la vie des nucléus (Valentin, Weber et Bodu 2014). En stade d'abandon, ces nucléus peuvent donc être de morphologies assez variables en fonction de la morphologie initiale du support et du degré d'exhaustion du nucléus (fig, 11a). Les plus typiques pourraient être classifiés typologiquement comme grattoirs épais circulaires ou semi-circulaires, tandis que les moins typiques peuvent être classifiés comme des grattoirs ou racloirs épais sur éclat ou bloc, ou même des grattoirs épais doubles sur éclat laminaire. Au sein de la série, il existe également trois nucléus qui se rapprochent morphologiquement du grattoir caréné de l'Aurignacien ancien. Toutefois, ces nucléus ont produit, au moins dans leur dernière génération, des supports adaptés à une production de grattoirs Caminade.

\subsection{2 - Normes des supports et de la retouche des grattoirs Caminade}

Les produits recherchés sont des éclats lamellaires/courtes lamelles, souvent très légèrement réfléchis, entre 6,6 et $22 \mathrm{~mm}$ de long (10,2 mm en moyenne), 3,8 à 9,3 de large (6,2 $\mathrm{mm}$ en moyenne), et ayant une épaisseur entre 0,6 et 2,6 mm (1,4 mm en moyenne) (fig. 13). Les produits sont le plus souvent de profil très légèrement courbe, parfois rectiligne, à bords plutôt parallèles (avant la retouche) et ils peuvent avoir des nervures subparallèles à légèrement convergentes sur la face supérieure. L'étude de la chronologie des enlèvements antérieurs sur les 54 grattoirs Caminade et pièces semblables ${ }^{5}$ (26 grattoirs Caminade, 22 grattoirs Caminade atypiques et 6 grattoirs Caminade assimilés) à Brignol indique que si des nervures convergentes ne sont pas déjà présentes, de très courts enlèvements, qui interviennent probablement au moment de l'abrasion du bord du plan de frappe, semblent mettre en place cette convergence souhaitée. Si dans la majorité des cas une telle recherche de nervures convergentes, souvent associée également à des nucléus à flancs convergents, relève d'une intention de produire des éléments naturellement appointés, les supports de grattoirs Caminade sont un contre-exemple. II apparaît que cette convergence de nervures, couplée avec une table trapézoïdale n'ayant pas une partie distale convergente, permet de contrôler le moment de terminaison des enlèvements et ainsi la longueur des produits, tout en ayant des parties proximales relativement larges et des parties distales non appointées. La préparation soigneuse du point d'impact par abrasion et la présence de nervures subparallèles à légèrement convergentes servent à guider très précisément l'initialisation de l'onde de choc, et ainsi donnent une morphologie convexe symétrique, souvent en forme de « $U$ », à la partie proximale des supports. Tout cela révèle une forte prédétermination et calibration des supports, même avant leur retouche. Plusieurs pièces brutes ayant les mêmes critères morphologiques que les grattoirs Caminade, et utilisées de la même manière

(5) À savoir les produits atypiques et les produits bruts (les grattoirs Caminade " assimilés ") ayant la même morphologie que les produits retouchés et portant les mêmes traces. 


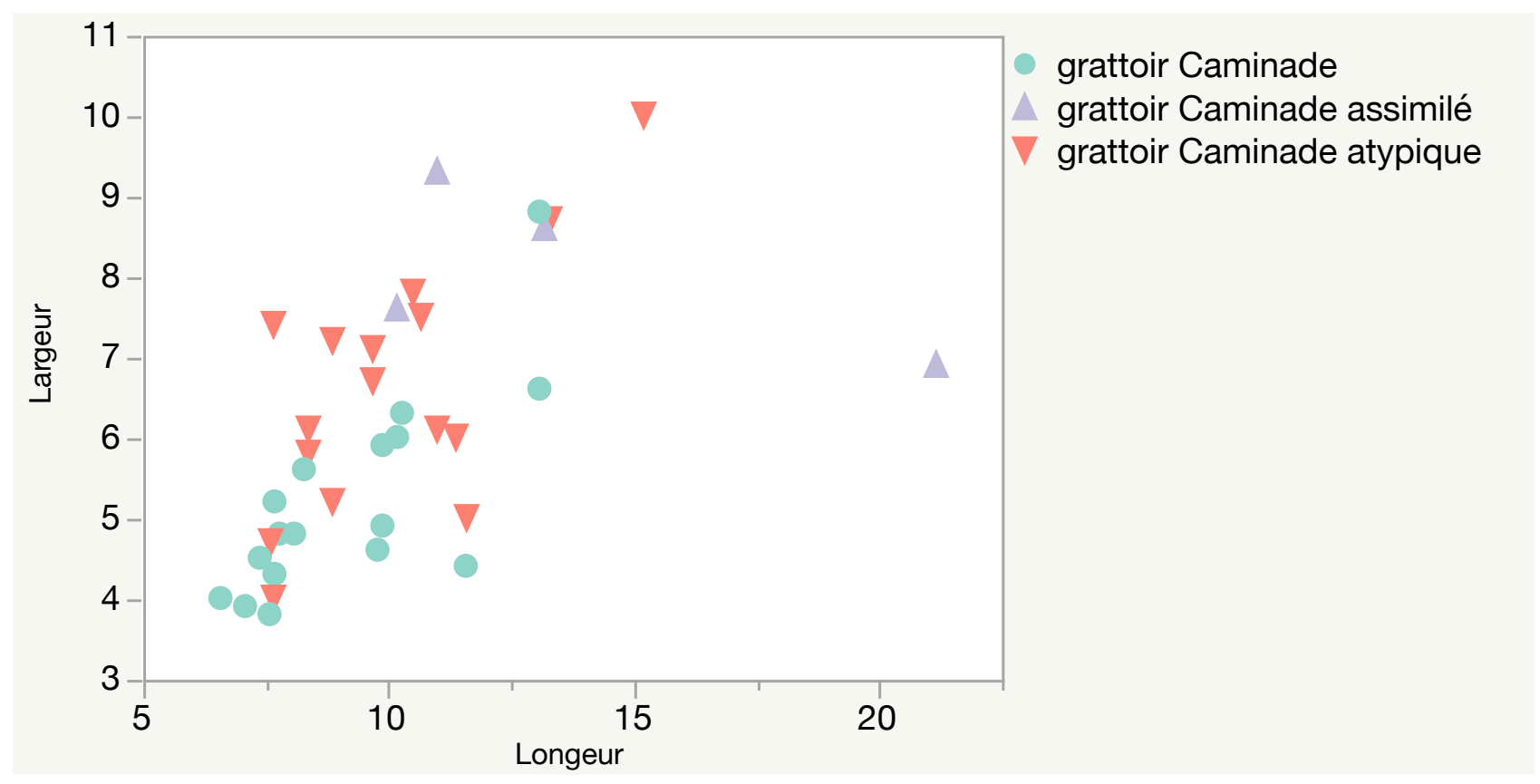

Figure 13 - Longueur $x$ Largeur (en mm) des grattoirs Caminade entiers typiques $(n=17)$, atypiques $(n=16)$ et assimilés $(n=4)$ (L. Anderson).

Figure 13 - Length by width (in $\mathrm{mm}$ ) of whole typical $(n=17)$, atypical $(n=17)$, and analogous $(n=4)$ grattoirs Caminade $(L$. Anderson).

(cf. infra, étude tracéologique), soutiennent cette hypothèse de calibration et prédétermination dès la phase de débitage.

Parmi les 54 pièces étudiées (fig. 14), $59 \%(n=32)$ des produits portent une troncature inverse sur la partie distale de la pièce, ou des retouches inverses partielles qui évoquent une même fonction que cette dernière. Cette troncature est souvent convexe, mais aussi asymétrique, car elle déborde fréquemment sur le bord droit du support $(n=21)$. Dans de plus rares cas, elle est latéralisée vers le bord gauche $(n=5)$, ou même symétrique $(n=6)$. Neuf éléments portent des retouches directes en parties distales qui évoquent un front de grattoir.

Le bord gauche porte souvent des retouches directes continues ou partielles $(n=34)$ qui ont pour but la régularisation de celui-ci. Quand le bord gauche ne porte pas de retouche, il est déjà relativement rectiligne (14 des 18 cas).

Le bord droit est le plus souvent laissé brut, mais la modification la plus courante $(n=18)$ est une retouche inverse continue ou partielle qui est en réalité la suite de la troncature inverse. Le rôle de cette retouche est sans doute l'atténuation de l'angle entre le bord droit et la partie distale de la pièce.

Enfin, la partie proximale est souvent laissée brute $(n=24)$, mais quand elle porte des retouches elles sont directes et donnent un aspect de front de grattoir. Ce front peut être symétrique $(\mathrm{n}=9)$, mais aussi asymétrique, surtout vers le bord droit $(n=10)$.
Si la méthode de production des grattoirs Caminade a comme objectif un produit déjà très calibré, les retouches jouent un rôle similaire à savoir la création d'une armature modulaire très standardisée. La retouche directe, majoritairement sur le bord gauche, crée un dos semiabrupt relativement rectiligne qui s'oppose à un bord convexe brut ou modifié par une retouche qui atténue les intersections entre le bord droit et les extrémités et qui accentue l'aspect convexe de ce dernier. Le résultat est un produit modulaire ayant souvent une morphologie en « $D$ » majuscule (une norme morphologique déjà évoquée par J. Pelegrin, com. pers.).

La hiérarchisation des modifications nous permet de discuter de la fonction de la retouche, ainsi que de ses implications pour l'emmanchement. Tout d'abord, la troncature inverse est très courante, tandis que la reprise du talon est plus rare. Nous imaginons, dans l'hypothèse d'emmanchement latéral déjà évoquée à plusieurs reprises (Sonneville-Bordes et Mortureux 1955 ; Morala, Lenoir et Turq ; Caux 2015), que ces modifications des extrémités pourraient avoir comme but une régularisation pour faciliter le chevauchement des pièces dans la hampe, ce qui impliquerait un tranchant long constitué de plusieurs de ces éléments modulaires. Nous avons déjà soulevé l'idée que la préparation spécifique des supports de grattoir Caminade a pour but le contrôle de la terminaison distale, ainsi que l'attribution d'une morphologie en « $U$ » à la partie proximale des pièces. Malgré cet essai de prédétermination, les terminaisons sont plus difficiles à contrôler que l'initiation de la fracture conchoïdale et nécessitent donc une reprise par troncature. Or, la 


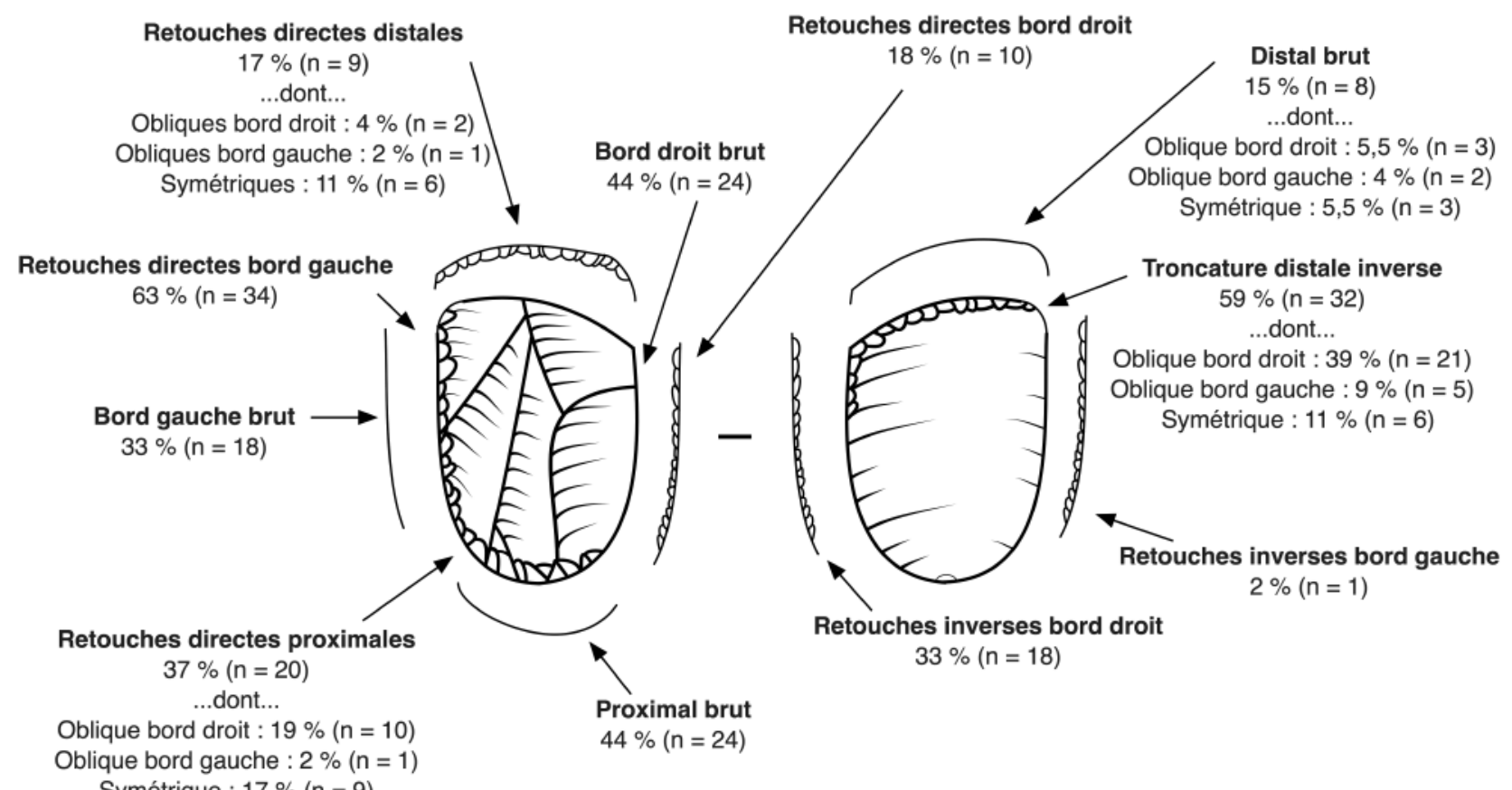

Figure 14 - Normes de retouches des grattoirs Caminade typiques $(n=26)$, atypiques $(n=22)$ et assimilés $(n=6)$, (proportions de parties fracturées non-figurées) (L. Anderson).

Figure 14 - Retouch norms for typical $(n=26)$, atypical $(n=22)$, and analogous grattoirs "Caminade " ( $n=6)$, (proportions of broken areas not represented) (L. Anderson).

régularisation des talons, déjà très standardisés à l'état brut, est moins fréquente. Enfin, les bords majoritairement latéralisés, avec un dos sur le bord gauche et des modifications pour accentuer l'aspect convexe du bord droit, nous apparaissent également comme des interventions pour faciliter un emmanchement latéral. En effet, les grattoirs Caminade atypiques ou assimilés, à savoir les produits qui s'écartent des normes de retouches décrites ci-dessus ou qui sont utilisés à l'état brut, renforcent l'idée que la forme souhaitée se rapproche d'un « D » majuscule. Les produits bruts portant des traces semblables à celles des véritables grattoirs Caminade ont souvent naturellement cette morphologie (fig. 15b). De plus, les objets atypiques démontrant une latéralisation inverse aux normes déjà décrites présentent souvent l'intention d'adapter cette forme de « $D$ » majuscule à des supports plus irréguliers (fig. 15c). Bien que ces exemples bruts ou atypiques soient peu nombreux dans la série, ils évoquent malgré tout la même conception morphologique que les grattoirs Caminade et portent les mêmes types de traces (cf. infra). Ainsi, nous devrions les inclure dans la même classe technofonctionelle.

\subsection{3 - Représentativité du schéma de Brignol au sein des autres séries à grattoirs Caminade}

Comme déjà évoqué, plusieurs études de la production des grattoirs Caminade furent réalisées (Caux 2015 ; Morala,
Lenoir et Turq 2005). Nous effectuerons donc ici une brève comparaison avec ces dernières pour évaluer la représentativité du schéma opératoire mis en évidence à Brignol.

La variabilité morphométrique des supports sélectionnés à Brignol pour être transformés en grattoirs Caminade rentre bel et bien dans la variabilité connue pour d'autres sites (Le Pigeonnier : Morala, Lenoir et Turq 2005 ; Caux 2015), Le Flageolet, la Grotte XVI, Maldidier et Caminade : Caux 2015). Cette variabilité morphométrique semble être continue et ne dépasse pas la longueur maximale des tables lamellaires concernées. Une seule différence à noter est l'absence de grattoirs Caminade de grande taille (supérieure à $25 \mathrm{~mm}$ ), mis en évidence au Flageolet (Rigaud 1982 ; Caux 2015). II est intéressant de noter que, même si de tels produits ne se présentent pas dans l'ensemble de Brignol, trois nucléus carénés ayant produit des grattoirs Caminade de taille normale dans leur dernière génération, ont des tables lamellaires entre 22,1 et $33,8 \mathrm{~mm}$, et auraient donc pu produire des supports pour ces éléments de grande taille. Le fait que les talons de ces grands supports témoignent d'un même traitement que les produits plus petits, et que la morphologie distale de ces éléments évoque celle des éclats lamellaires plus réduits (Caux 2015), renforcent cette idée. Bien que les nucléus à grattoir Caminade de morphologie grattoir caréné de Brignol n'aient pas pu produire les plus grands éléments du 

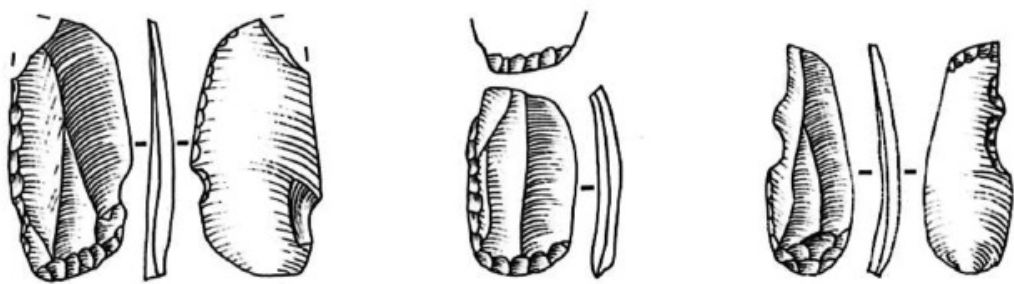

$2 \mathrm{~cm}$

$\uparrow$

$千$

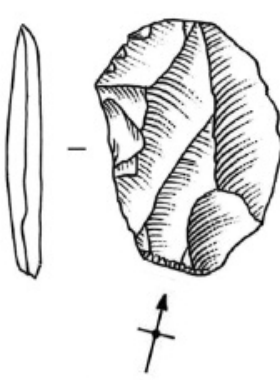

b

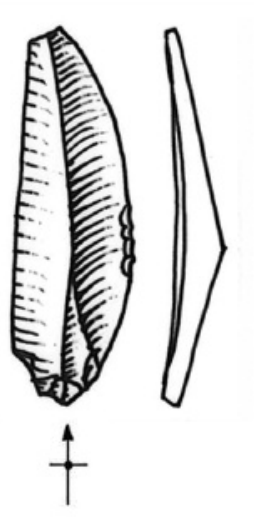

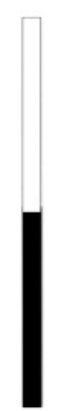

Figure 15 - Grattoirs Caminade typiques (a), assimilés (bruts) (b) et atypique (c). En inversant le grattoir Caminade atypique (c), tous les exemples figurés démontrent l'intention de produire une armature en forme de " $D$ » majuscule (Dessins $R$. Picavet).

Figure 15 - Typical (a), analogous (unretouched) (b), and atypical (c) grattoirs Caminade. By inverting the atypical grattoir Caminade (c) all examples figures show the intention of producing an armature with a capital « $D$ » morphology (Drawings $R$. Picavet).

Flageolet (44 mm) (Caux 2015), ils auraient pu produire une partie de ce regroupement. Cela nous permet d'étayer le scénario d'une certaine homogénéité dans la production des différents gabarits d'éclats lamellaires, avancé par S. Caux (2015), et d'émettre l'hypothèse selon laquelle la production des grattoirs Caminade s'effectue largement sur des nucléus de type grattoir épais circulaire/subcirculaire. Pour les grattoirs Caminade de plus grands calibres, ils sont peut-être produits depuis des nucléus plus atypiques de type "grattoir caréné », qui peuvent tout de même produire, de manière intercalée, l'intégralité des gabarits des grattoirs Caminade.

En ce qui concerne la morphologie initiale des produits souhaités, malgré quelques différences de proportions et de vocabulaire, il apparaît que la recherche des éclats lamellaires à bords subparallèles, voire convexe sur au moins un bord, avec des nervures dorsales subparallèles (Morala, Lenoir et Turq 2005) à légèrement convergentes (Caux 2015), conditionne le schéma opératoire de la production des grattoirs Caminade.

Le contrôle de la longueur des supports semble montrer plus de variabilité que leur morphologie générale. À Brignol, il apparaît que plusieurs éléments contribuent au contrôle intentionnel de la longueur des supports (fig. 11). Au Pigeonnier, le seul autre site sur lequel une étude systématique des nucléus fut menée, il apparaît, qu'à l'état d'abandon au moins, la longueur des supports est contrôlée par l'épaisseur du nucléus et donc la longueur de la table lamellaire elle-même (Morala, Lenoir et Turq 2005). De plus, des amincissements des supports-nucléus, sans doute pour calibrer la longueur des tables lamellaires, ont été observés au Pigeonnier (Morala, Lenoir et Turq 2005), tandis que cette modalité de contrôle de la longueur de la table lamellaire n'existe pas à Brignol. L'inverse par contre, à savoir la présence des modalités d'entretien (éclats lamellaires de nettoyage) et de contrôle de la longueur des supports (convergence des nervures et table non convergente en partie distale) mis en évidence à Brignol, dans l'ensemble du Pigeonnier, ou d'autres ensembles, reste à explorer.

Une autre différence concerne l'angle de débitage, majoritairement vers $60^{\circ}$ dans l'ensemble des nucléus à Brignol, mais vers $45^{\circ}$ au Pigeonnier (Morala, Lenoir et Turq 2005). Cela pourrait être expliqué par des différences de support initial et des contraintes différentes liées à la 
variabilité des matières premières, majoritairement des éclats épais ou des fragments de bloc à Brignol, contre des lames et des éclats épais au Pigeonnier. Un nucléus atypique s'écarte fortement de la norme à Brignol et présente ce même angle de débitage à $45^{\circ}$, ce qui pourrait évoquer des différences idiosyncrasiques entre tailleurs ou par rapport au support initial. II faut tout de même noter que les supports-nucléus moins épais présentent souvent un angle de débitage vers $45^{\circ}$ qui imite parfaitement l'angulation du front des véritables grattoirs du fond commun domestique, et donc il est fort possible qu'il existe un certain recouvrement entre ces deux catégories (une idée en partie soutenue par les données tracéologiques cidessous). Cela pourrait être soit le reflet d'une réelle fluidité technofonctionelle ou morphologique de la part des préhistoriques, soit l'effet de nos propres outils analytiques, trop rigides pour traiter de manière adéquate la flexibilité des gestes préhistoriques.

Le détachement des supports dans toutes les séries connues à ce jour est précédé par la préparation systématique de la corniche de l'unique plan de frappe, par abrasion, ce qui donne un aspect " front de grattoir » aux talons bruts, déjà très standardisés (Morala, Lenoir et Turq 2005 ; Caux 2015). Quand les talons sont lisibles et non recoupés entièrement par la retouche, du lisse-abrasé, voire parfois même du punctiforme-abrasé (Caux 2015), parfois associé avec de très petites lèvres (Morala, Lenoir et Turq 2005), dominent les ensembles. Ces stigmates, comme on l'a déjà vu à Brignol, sont compatibles avec l'utilisation d'un percuteur tendre organique relativement dense, et ont été recréés expérimentalement (Morala, Lenoir et Turq 2005). Toutefois, nous signalons qu'il y a un manque important d'expérimentation concernant la diversité des techniques de détachement pour les industries lamellaires. La production de grattoirs Caminade par d'autres techniques, non testées expérimentalement, est donc une hypothèse à ne pas écarter entièrement.

Quant à la morphologie imposée par la retouche des produits, les mêmes tendances, avec une hiérarchisation des interventions semblable, se dégagent à Brignol et les autres séries à grattoirs Caminade. Un bord gauche rectiligne, parfois régularisé par retouche directe, qui s'oppose à un bord droit convexe brut est le trait le plus systématique dans l'intégralité des séries (Morala, Lenoir et Turq 2005 ; Caux 2015). Ensuite, la troncature inverse distale, qui a tendance à déborder inversement sur le bord droit et donc à accentuer son aspect convexe, permet de régulariser la partie distale des supports (Morala, Lenoir et Turq 2005 ; Caux 2015). Enfin, la reprise du talon par retouche directe convexe, en morphologie de « front de grattoir ", semble être l'intervention la moins systématique. Cela est certainement dû à une préparation soigneuse des corniches préalablement au détachement des supports (Morala, Lenoir et Turq 2005) qui éviterait de régulariser la partie proximale dans un second temps.

Notons que les proportions (fig. 14) des types de traitements des bords à Brignol se distinguent nettement de ceux rapportés antérieurement (Caux 2015). II est possible que cela soit lié à l'échantillonnage des séries. Dans notre étude, nous avons pu intégrer les produits atypiques et bruts ayant les mêmes traces et les mêmes critères morphologiques que les grattoirs Caminade typiques, ce qui n'est pas toujours le cas pour d'autres études.

Quoi qu'il en soit, la hiérarchisation des critères morphologiques des grattoirs Caminade semble démontrer une intention partagée entre toutes les séries, la recherche d'une armature modulaire ayant une morphologie qui se rapproche d'un « $D$ » majuscule, ce qui doit sans doute être mis en lien avec un mode d'emmanchement partagé par l'ensemble des séries connues à ce jour. II apparaît également que, malgré quelques petites divergences, le schéma opératoire de production des grattoirs Caminade dégagé à Brignol est, a priori, compatible avec le schéma déjà décrit au Pigeonnier (Morala, Lenoir et Turq 2005), ainsi que pour les produits décrits au Flageolet, à Caminade, au Pigeonnier, à Maldidier et à la Grotte XVI (Caux 2015).

\section{5 - Analyse fonctionnelle de l'assemblage}

La question de l'utilisation des grattoirs Caminade est donc centrale. Plus largement, il nous a paru particulièrement intéressant de nous interroger sur la destination fonctionnelle de l'ensemble de l'outillage microlithique sur lamelles et éclats lamellaires dont font partie les grattoirs Caminade. Quel est le rôle de ces objets issus d'une chaîne opératoire bien distincte de celles des autres supports de l'outillage du site ? Pour comparaison, il était primordial de prendre également en compte le reste de l'outillage sur lames et sur éclats qui, a contrario des lamelles et éclats lamellaires, n'ont, en majorité, pas été produits sur place. La question de l'utilisation des grattoirs-nucléus après débitage a été également explorée.

\section{1 - Protocoles d'observation et d'analyse}

L'observation a été conduite à deux échelles (macroscopique et microscopique), selon des protocoles couramment utilisés en tracéologie (Semenov 1964 ; Keeley 1980 ; Plisson 1985), et en utilisant une base de référentiels expérimentaux de fabrication et d'utilisation couvrant le champ d'usage d'un vaste registre d'outils (projectile, grattoirs, burins, lames, lamelles, éclats, etc., ayant servi sur des matières d'œuvre variées de type peau, matières osseuses et carnées, végétales, etc., selon des gestes et des temps d'utilisation très divers), conservée au CEPAM (Nice).

L'observation macroscopique s'est révélée fructueuse, tandis que l'observation microscopique était rendue très difficile, voire impossible, par une altération mécanique et physico-chimique de la microtopographie du silex confirmée par l'analyse des états de surface (cf. supra). En effet, les pièces ont vraisemblablement subi un déplacement au sein du sédiment, altérant mécaniquement 
l'état de la microsurface du silex (présence d'un lustré de miroir).

Notons que notre observation microscopique ne s'est pas portée uniquement sur les bords, mais sur l'ensemble des surfaces de l'outil. Ainsi une bonne part des microtraces identifiées sur les bords était également visible au centre de la pièce témoignant sans conteste de leur origine taphonomique.

\section{2 - Les grattoirs Caminade}

Nous avons observé un échantillon de dix-neuf grattoirs Caminade selon le protocole susmentionné.

Leurs dimensions particulièrement réduites ont laissé penser que leur emmanchement conditionnait leur utilisation. Ainsi, les grattoirs Caminade serviraient probablement en armatures (Mortureux et SonnevilleBordes 1955 ; Morala, Lenoir et Turq 2005), soit emmanchées sur un outil ayant servi en percussion posée (type couteaux, scies, etc.), soit sur un outil ayant servi en percussion lancée (projectile de type sagaies ou flèches).

Nous avons donc recherché les traces pouvant témoigner de leur utilisation en tant qu'armatures de projectile. Les traces alors attendues sont des fractures transversales par flexion à languette supérieure ou égale à $2 \mathrm{~mm}$ de long, des fractures burinantes dont l'amplitude est supérieure ou égale à $2 \mathrm{~mm}$ et des microstries linéaires d'impact (notamment Moss et Newcomer 1982 ; Fischer, Vemming Hansen et Rasmussen 1984 ; Geneste et Plisson 1986 ; Crombé et al. 2001 ; Philibert 2002 ; O'Farrell 2004, 2005 ; Pétillon et al. 2011 ; Chesnaux 2013, 2014).

Néanmoins, aucun grattoir Caminade ne porte de telles traces. Seuls deux (nos 78 et 709) sont fracturés transversalement, mais de manière nette. Ce type de fracture est équivoque et a pu survenir au cours des processus de façonnage, d'utilisation ou encore postdépositionnels.

Leur morphologie non appointée et la présence de bord(s) tranchant(s) pourraient nous laisser penser que si ces objets avaient été utilisés comme armatures de projectile, ils auraient été emmanchés latéralement et non en tête de hampe, encore une notion déjà évoquée plusieurs fois (Morala, Lenoir et Turq 2005 ; Caux 2015). Les armatures de projectile emmanchées latéralement réagissent à l'impact de manière très différente de celles emmanchées en tête de hampe (Chesnaux 2014). En effet, elles sont bien moins exposées au choc que ces dernières et sont donc moins souvent et moins violemment endommagées, c'est-à-dire qu'elles présentent très rarement des fractures transversales. Par contre, on note une fréquence plus importante de fractures burinantes liées à l'entrechoquement des armatures entre elles. Des ébréchures sont également présentes, elles sont alors unidirectionnelles, perpendiculaires ou obliques à initiation en flexion ou en cône et à terminaison le plus souvent en charnière ou en gradin (Gassin 1996).

Les grattoirs Caminade ne présentent aucune fracture burinante. Ils portent par contre très fréquemment des enlèvements le long des bords. Néanmoins, ceux-ci ne présentent pas les caractéristiques des ébréchures d'impact. Ce sont, en effet, des esquillements isolés discontinus à initiation en cône et en flexion à terminaison lisse ou en marche (fig. 16-1), associés à des émoussés ponctuels et des micropolis orientés longitudinalement, non interprétables. Ces traces témoigneraient ainsi davantage de leur utilisation en coupe longitudinale sur matériau midur et légèrement abrasif ${ }^{6}$.

\section{3 - Les éclats lamellaires et lamelles bruts et retouchés}

Parmi les 121 éclats lamellaires et lamelles bruts et retouchés issus des mêmes séquences de débitage que les supports des grattoirs Caminade, 56 portent les mêmes types de traces que les grattoirs Caminade à savoir des ébréchures peu invasives, irrégulières et des bords émoussés à légèrement doucis (fig. 16-2).

Aucun ne présente de traces d'impact. Trente-trois (dont 25 lamelles brutes, deux lamelles retouchées et six éclats lamellaires bruts) sont fracturés transversalement de manière nette, 11 (sept lamelles brutes, trois éclats lamellaires bruts et un éclat lamellaire retouché) sont fracturés transversalement avec languette non diagnostique et une lamelle est fracturée de manière burinante non diagnostique.

Il est donc très probable que ces objets aient fonctionné de la même manière que les grattoirs Caminade à savoir en coupe longitudinale sur matériau mi-dur et légèrement abrasif. La retouche des grattoirs Caminade ne serait alors qu'une variable d'ajustement à la normalisation des formes de ces armatures destinées à s'insérer dans un même manche, ce qui est conforme avec l'étude des normes de retouches (cf. supra).

Étant donné les traces observées, nous pouvons émettre deux hypothèses quant à l'usage de ces objets et des grattoirs Caminade : soit une utilisation en armature de couteau pour une découpe fine de viande (Plisson 1985 ; Gassin 1996 ; Claud 2008) pour le séchage par exemple, soit une utilisation en armature de couteau pour le traitement des poissons (écaillage, découpe ou encore levée de filet, cf. Claud 2008). Ces deux hypothèses seront prochainement testées expérimentalement. Étant donné

(6) II est à noter également que H. Plisson avait relevé un unique indice d'usage sur les grattoirs Caminade du Flageolet (Plisson, non publié) à savoir de fines ébréchures témoignant d'une action longitudinale " contre matériau tendre avec contact occasionnel contre matériau dur (...) majoritairement sur le tranchant rectiligne des objets " (7 objets avec véritables macro-traces, 7 avec macro-traces douteuses). 

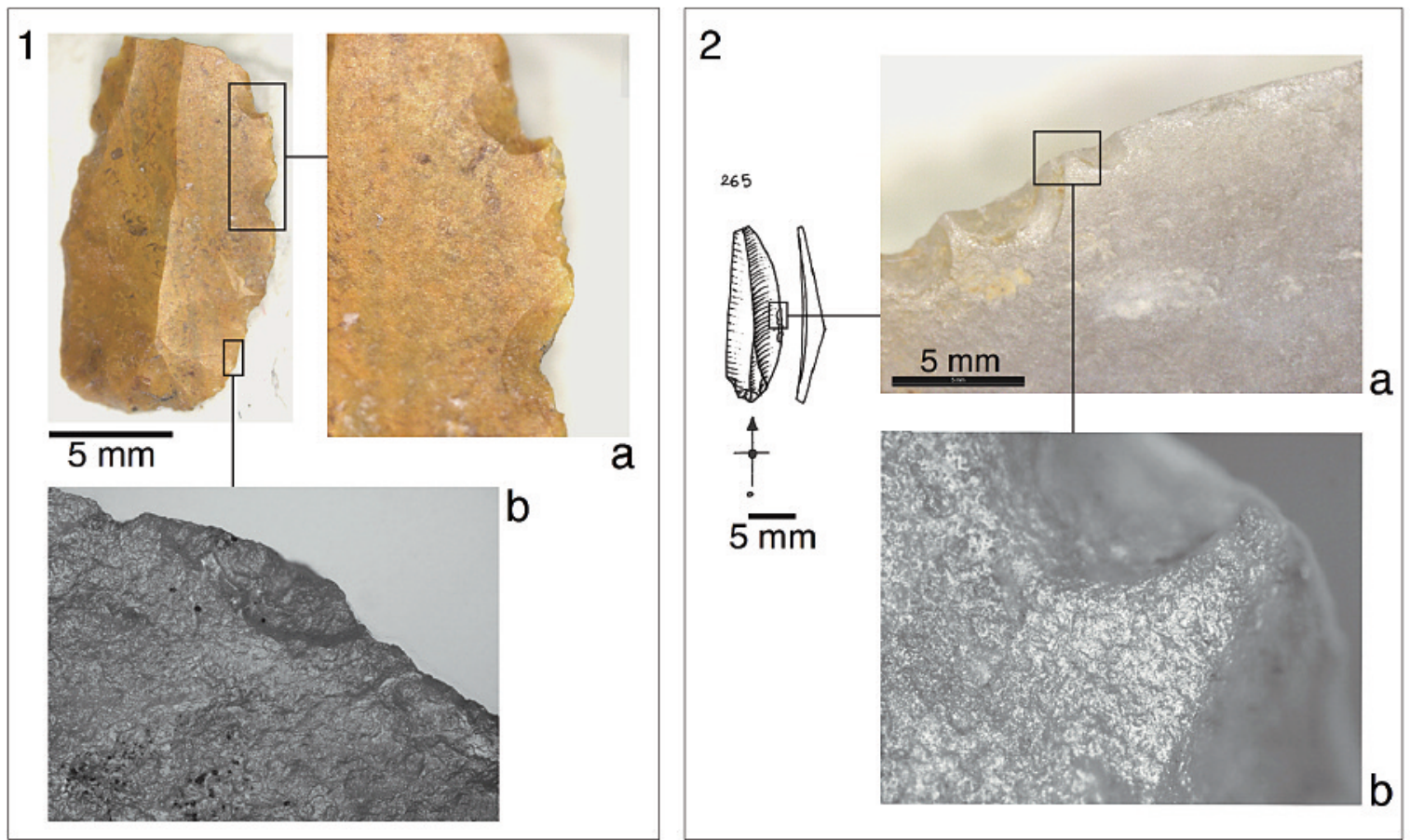

Figure 16 - 1 : Grattoir Caminade $n^{\circ} 701$ en silex d'aspect jaspéroïde ; a. Détail du bord ébréché ; $b$. Détail de la microsurface altérée du silex (x 200). 2 : Lamelle brute $n^{\circ} 265$; a. Détail du bord supérieur droit dont les ébréchures et le fil sont émoussés ; b. Détail x 200 du bord ébréché et émoussé associé à un micropoli longitudinal altéré (L. Chesnaux).

Figure 16 - 1: Grattoir Caminade $n^{\circ} 701$ in jasperoid-like flint; a. chipped edge; b. altered microsurface (x 200). 2: Unretouched bladelet $n^{\circ} 265$; a. right edge dorsal surface showing rounding of the chipped areas and the cutting edge; chipped edge and edge rounding associated with a longitudinal altered micropolish (x 200) (L. Chesnaux).

les dimensions particulièrement réduites de ces armatures, une utilisation pour la découpe ou encore la désarticulation des carcasses est, d'après nous, à exclure.

\section{4 - Les nucléus}

Nous avons analysé les dix nucléus liés à une production de grattoirs Caminade. Ces objets sont bien des nucléus comme le montrent les remontages -, mais ils ont également, dans un deuxième temps, servi pour quatre d'entre eux ( $\mathrm{n}^{\mathrm{OS}} 284,374,658$ et 341 ) au grattage d'un matériau tendre (absence d'ébréchures) abrasif (émoussé), peut-être de type peau fraîche. Les émoussés transversaux au bord et visibles à l'interface entre le plan de frappe et la table lamellaire sont de degrés divers selon les pièces (fig. 17). Ils varient de peu intenses ( $\left.n^{\circ} 284\right)$ à assez intenses $\left(n^{\circ}\right.$ 341). Quelques stries sont également présentes.

\section{5 - Le reste de l'outillage : les lames (dont les grattoirs sur lames) et les éclats retouchés}

S'il est possible de se prononcer sur l'utilisation de la production lamellaire - étant donné le grand nombre de produits et la récurrence des traces macroscopiques malgré la piètre conservation des micropolis, il est particulièrement délicat de proposer une reconstitution de la fonction et du fonctionnement du reste de l'outillage sur lames et sur éclats. Plusieurs zones potentiellement utilisées ont, malgré tout, été identifiées.

Ainsi parmi les quatre grattoirs analysés ( $n^{\text {os }} 495,365$, 28_inrap et 361), nous avons observé deux fronts présentant pour l'un un émoussé peu intense ( $n^{\circ} 28$ inrap) et pour l'autre plus marqué ( $\left.n^{\circ} 495\right)$. Ils se répartissent tous les deux de manière transversale au fil témoignant d'un geste de raclage sur un matériau tendre (quasi absence d'esquillements) abrasif (présence d'un émoussé), certainement de type peau (tout comme les nucléus).

Deux autres objets présentent une zone utilisée assez explicite. Ce sont deux éclats retouchés et émoussés de manière très localisée ( $n^{\text {os }} 134$ et 643 ). La face inverse du bord distal rebroussé de l'éclat 134 est retouchée et fortement ébréchée. Les ébréchures et retouches abruptes à semi-abruptes sont légèrement émoussées dans leur totalité, perpendiculairement au bord. Le bord distal de l'éclat 134 a donc servi à racler un matériau dur abrasif en coupe négative. En l'absence de micropoli identifiable, il est impossible de se prononcer plus précisément sur le matériau travaillé. La face supérieure du bord gauche distal de l'éclat 643 porte également un émoussé perpendiculaire au bord. Celui-ci est assez intense et n'est associé à 


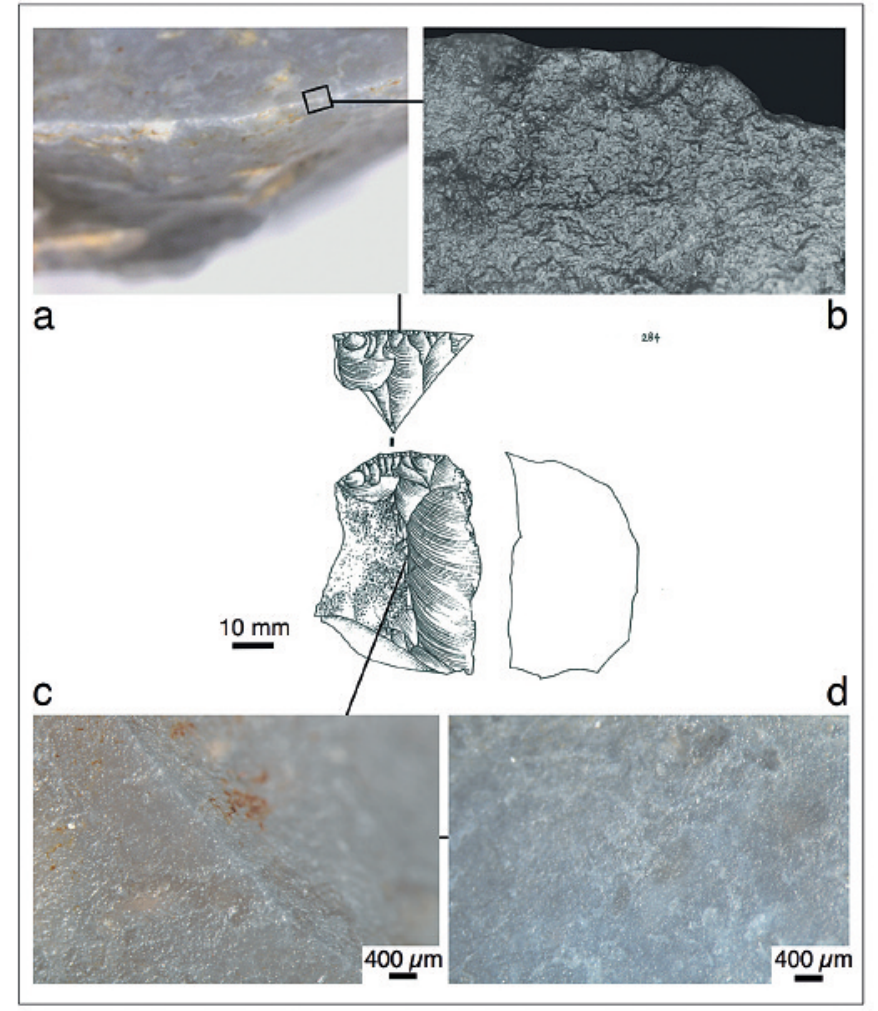

aucune ébréchure. Ce bord a donc servi au raclage d'un matériau souple et abrasif.

\section{6 - Conclusion}

Malgré la piètre conservation de la microtopographie des silex, nous avons montré, grâce aux traces macroscopiques, que la production lamellaire n'était pas dévolue à l'armement, mais certainement à un travail de type découpe de viande ou pourquoi pas au traitement de poissons. Quant à sa participation à la sphère domestique ou cynégétique, l'attribution s'avère délicate et étroitement liée à la fonction du site et à la segmentation des chaînes de traitement dans le temps et dans l'espace. II s'avère, de plus, que plusieurs nucléus à lamelles ont été utilisés comme grattoir après la production lamellaire.

\section{6 - Synthèse et conclusions}

Brignol offre, pour la première fois pour une série de l'Aurignacien récent à grattoirs Caminade, une lecture croisée de données technoéconomiques, technofonctionnelles, pétroarchéologiques et géoarchéologiques. Le résultat de cette vision assez complète nous permet d'avancer des hypothèses fonctionnelles à l'égard des « fameux " grattoirs Caminade, et d'évoquer quelques interprétations provisoires liées à la fonction du site et au statut de ce faciès particulier au sein de l'Aurignacien récent.

Dans un premier temps, l'étude techno-économique de la série, ainsi que la recherche et l'analyse poussées des remontages, nous permettent d'étayer l'hypothèse d'une
Figure 17 - Nucléus à grattoirs Caminade (type « grattoir caréné ") n०284; a. Détail du bord de plan de frappe légèrement émoussé par une action transversale; $b$. Vue du bord émoussé x 200 et de l'altération de la microtopographie du silex; c. Arête de la face supérieure recouverte d'un lustré de miroir et ne présentant pas d'esquillement ; $d$. Détail de la face inférieure recouvert d'un voile blanchâtre (L. Chesnaux).

Figure 17 - Grattoirs Caminade core ("carinated scraper" type) $n^{\circ} 284$; a. edge of striking platform showing light edge rounding resulting from a transverse action; $b .200 x$ magnification of rounded edge and flint's altered microtopography; $c$. arris on dorsal surface covered by a mirror lustre showing no splintering; $d$. ventral surface showing initial formation of white patina (L. Chesnaux).

certaine unité technologique au sein des séries à grattoirs Caminade. Les normes de retouches et de sélection des supports sont quasi-identiques entre Brignol et les autres séries connues (Morala, Lenoir et Turq 2005 ; Caux 2015). Enfin le schéma opératoire complet décrit à Brignol, malgré quelques possibles particularités, est a priori similaire aux productions observées ailleurs.

L'étude tracéologique souligne une certaine complexité à l'égard du statut de ces grattoirs Caminade, à mettre en lien sans doute avec le statut du site et peut-être le faciès technologique lui-même. Bien que le microlithisme aurignacien ait souvent été attribué à la sphère cynégétique (Bon 2005) dans le cadre de la production d'armatures destinées spécifiquement aux armes de jet (O’Farrell 2005 ; Normand, O'Farrell et Rios Garaizar 2008), il apparaît qu'une telle utilisation est à écarter pour les microlithes de type grattoir Caminade en faveur d'une fonction de type couteau modulaire. Cette hypothèse a d'ailleurs déjà été évoquée par des études tracéologique (Plisson, non publié) et technologique (Caux 2015) antérieures de la série du Flageolet et de Caminade.

La hiérarchisation des différents types de modifications des produits (cf. supra) nous permet d'avancer plusieurs hypothèses d'emmanchement à tester prochainement au moyen d'expérimentations (fig. 18). Bien qu'aucune trace ne se présente sur les parties proximales ou distales des objets étudiés, la relation systématique entre le bord droit, souvent convexe et brut, et le bord gauche, souvent rendu droit par une retouche directe, reste difficile à interpréter. L'étude de H. Plisson (non publiée) sur les 

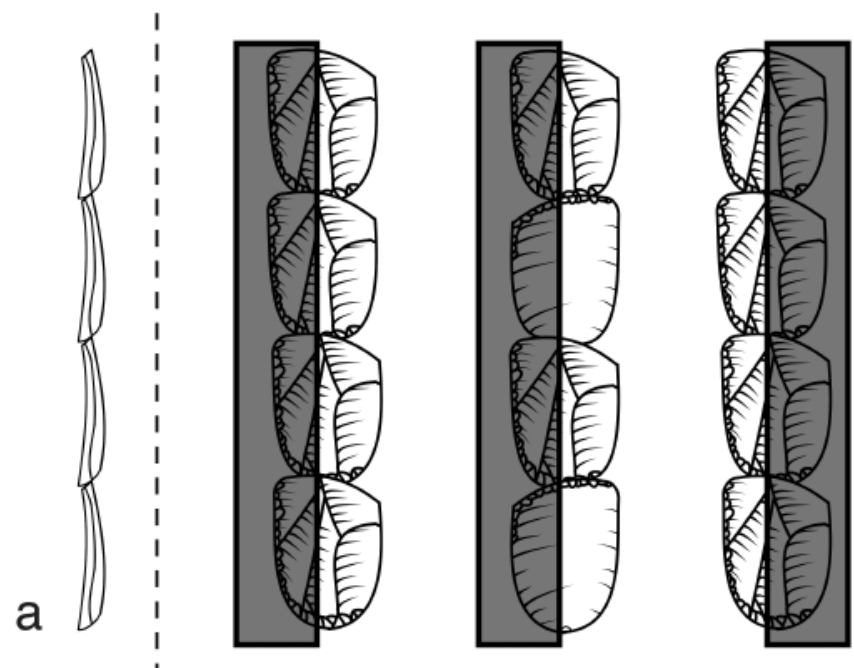

$\mathrm{b}$
Figure 18 - Hypothèses d'emmanchement des grattoirs Caminade : a. chevauchement hypothètique des armatures dans la hampe; b. quelques possibilités d'emmanchement latéral en série selon les normes de retouche des éléments et les données tracéologiques (L. Anderson).

Figure 18 - Grattoirs Caminade hafting hypotheses: a. hypothetical overlap of armatures in the haft; $b$. a few possibilities for the lateral hafting of elements in series according to retouch norms and use-wear data (L. Anderson). grattoirs Caminade du Flageolet a souligné une latéralisation préférentielle des traces sur « le tranchant rectiligne ", tandis que les traces ne sont pas particulièrement latéralisées dans l'ensemble étudié de Brignol. Au vu des tailles respectives des échantillons examinés, il est possible que l'ensemble du Flageolet ne soit pas statistiquement représentatif et donc pas réellement le reflet d'une certaine variabilité dans les techniques d'emmanchement. Pourtant cela ne répond pas à la question cruciale qui découle de ces observations (les normes de retouches des grattoirs Caminade sont latéralisées, mais les traces ne le sont pas) : quelle(s) fonction(s) et/ou technique(s) d'emmanchement pourraient expliquer cette divergence?

Un élément qui est partagé par les technologies lamellaires plus classiques de l'Aurignacien et celle des grattoirs Caminade semble bel et bien être cette notion de modularité - la possibilité de produire des éléments relativement standardisés, facilement remplaçables et avec un rendement élevé par rapport à la quantité de matière première nécessaire (Bon 2005). Ce dernier point implique également la possibilité que ces productions lamellaires soient étroitement liées à une stratégie de mobilité très flexible au sein de laquelle la dépendance aux sources de matières premières de grande qualité est particulièrement réduite.

Malgré ces similarités, les aspects fonctionnels du reste de l'assemblage et le mode de gestion des matières premières évoquent un statut particulier pour ce site. Ainsi, en l'absence de la faune, non conservée, la principale activité observable in situ est celle de la production des grattoirs Caminade. Ce constat est corroboré par la proportion la plus élevée de matières premières lointaines représentée par cette dernière. Cela renvoie à une anticipation des besoins liée à la technologie composite des grattoirs
Caminade et donc à une certaine spécialisation fonctionnelle du site. La forte proportion de quartz « culinaires » et la présence d'au moins un macro-outil sont peut-être à mettre en lien avec cette fonction spécifique. L'absence relative de production laminaire et d'outillage traditionnellement « domestique », ainsi que le recyclage de certains nucléus pour le traitement des peaux soulignent l'aspect secondaire de ces activités par rapport à celles liées plus étroitement à l'utilisation des grattoirs Caminade. Si l'on ajoute à cela la présence de savoir-faire moyens et compétents, mais pas de débitages très malhabiles, il est fort possible qu'une seule partie du groupe soit présente sur les lieux (Anderson, thèse en cours). Ainsi, même si une partie significative du site est manquante, nous pouvons émettre deux hypothèses : soit Brignol est un locus spécialisé d'un site plus important, soit c'est un site logistique de très courte durée où le rôle des grattoirs Caminade et accessoirement celui des quartz « culinaires » est primordial. Bien que nous ne puissions pas expliciter la réelle fonction du site, l'ensemble des résultats technologiques, pétroarchéologiques et tracéologiques, qui nous fournissent des informations sur le fonctionnement du lieu, témoignent d'un site ou d'un locus dont l'occupation est de courte durée et de fonction plutôt spécialisée.

Enfin, les traces observées témoigneraient d'un lieu de traitement de peau fraîche ou remouillée, mais surtout un travail de type boucherie relativement fin. L'emmanchement des grattoirs Caminade en série envisagé implique une hampe bien plus épaisse que le tranchant, ainsi qu'un tranchant peu large qui rentrerait très peu dans la matière travaillée. Ce type d'outil serait donc mal adapté aux premières étapes de traitement du gibier, mais pourrait ainsi être très utile pour la découpe de fines tranches de viande, peut-être destinées au stockage après séchage. 
Brignol peut-il nous aider à comprendre la relation entre le faciès aux grattoirs Caminade et les autres faciès de l'Aurignacien ? En l'état, il ne peut que suggérer de futures voies de recherche sur le sujet. L'aspect homogène de l'ensemble, la probabilité qu'il soit le résultat d'une seule occupation relativement brève et l'absence d'autres marqueurs culturels de l'Aurignacien récent sont de forts arguments pour l'unité de la technologie lithique du faciès à grattoirs Caminade. Sa relation avec les autres faciès reste néanmoins floue. Brignol nous paraît relativement spécialisé (ou au moins un locus spécialisé), et le fait qu'il s'agisse d'un site de plein air ne serait pas incompatible avec une complémentarité fonctionnelle liée aux différents contextes topographiques. Cela expliquerait les associations entre certains faciès de l'Aurignacien récent et les industries à grattoirs Caminade en contexte stratifié. Pourtant, pour l'instant ces associations restent délicates, et donc il est également possible que les industries à grattoirs Caminade soient bel et bien une phase proprement chronoculturelle et régionale de l'Aurignacien. Une réévaluation taphonomique de toutes les séries en question, une reprise technologique des autres types de production (laminaire, etc.) dans les sites à attribution culturelle unique (à savoir Le Pigeonnier, Maldidier et Las Pélénos 2), et une confrontation de ces données à celles qui existent déjà pour les différentes phases à grattoir à museau, burin busqué et burin busqué déstructuré, seront nécessaires pour réellement trancher ou simplement nuancer les deux hypothèses évoquées. Enfin, la découverte inédite par A. Morala sur le site de Fournol (Lot) d'une industrie à grattoirs Caminade, lors des fouilles menées sous sa direction, vont certainement apporter de nouvelles informations sur ce sujet.

Revenons enfin sur le statut spécifique de ces grattoirs Caminade. Appartiennent-ils à la sphère domestique ou cynégétique ? L'interprétation reste délicate en l'état de nos recherches. Des expérimentations, ainsi que l'exploration des notions évoquées dans cet article sur d'autres sites, seront nécessaires pour étayer les hypothèses fonctionnelles présentées. Un couteau de chasse participerait bel et bien à la sphère cynégétique, mais s'il est associé avec le traitement du gibier et des peaux sur un site spécialisé, où devrait-on établir la frontière entre ce qui est proprement domestique et ce qui est proprement cynégétique ? Bien qu'un outil heuristique très utile, cette division est sans doute une simplification de la réalité, et peut-être devrions-nous évaluer cet outil à l'aune d'autres critères, tels que la gestion des matières premières et la segmentation des chaînes lithiques et fauniques dans l'espace et les rôles relatifs des différents sites. Cela nous permettra d'avancer des interprétations sur les circulations des chasseurs-cueilleurs aurignaciens et les systèmes auxquels ils appartiennent au lieu de se reposer sur des dichotomies simplifiées, qui, si elles s'avèrent utiles dans un premier temps, contribuent par ailleurs à caricaturer une situation vraisemblablement plus complexe.

\section{Remerciements}

II nous est agréable de remercier ceux qui ont contribué au bon déroulement de la phase de terrain et qui ne figurent pas parmi les auteurs de cet article: A. Deville, W. Galin, I. Guerrero, G. Parpaite, J. Recchia et A. Roy. Cette fouille n'aurait pas vu le jour sans les implications du SRA Aquitaine, du Pôle de Santé du Villeneuvois et de Paléotime. Nous remercions également $M$. Lejay pour sa révision du texte et ses commentaires ainsi que les relecteurs sollicités par la revue.

\section{Références bibliographiques}

AIRVAUX J., BERTHET A.-L., CASTEL J.-C. 2003 - Le gisement aurignacien de Chez-Pinaud 2, à Jonzac, Charente-Maritime, Bulletin Préhistorien du Sud-Ouest, 10, 1, p. 25-75.

ANDERSON L., BON F., BORDES J.-G., PASQUINI A., SLIMAK L., TEYSSANDIER N. 2015 - Relier des espaces, construire de nouveaux réseaux : aux origines du Protoaurignacien et des débuts du Paléolithique supérieur en Europe occidentale, In : N. Naudinot, L. Meignen, D. Binder, et G. Querre (eds), Les systèmes de mobilité de la Préhistoire au Moyen Âge. XXXVe rencontres internationales d'archéologie et d'histoire d'Antibes, Antibes, Éditions APDCA, p. 93-109.

ANDERSON L., LEJAY M., BON F., MENSAN R., BRUGAL J.-Ph., COSTAMAGNO S., HECKEL C., ARAUJO M., MÉNARD M., PRADEAU J-.V., SALOMON H., SELLAMI F., BARSHAY-SZMIDT C. (en préparation) Insights into Aurignacian daily life and camp organization: the open-air site of Régismont-le-Haut.

BALLARIN C., BENQUET L. (de), BERTRAN P., CHOPIN J.-P., DUMAS A., FOURLOUBEY C., SIREIX C. 2011 - Aquitaine, Lot-et-Garonne, Villeneuve-sur-Lot Brignols, Romas, INRAP Grand Sud-Ouest, 122 p.

BATCHELOR D. 1979 - The Use of Quartz and Quartzite as Cooking Stones, In G. Bosinski (dir), Die ausgrabungen in Gönnersdorf - und die siedlungsbefunde der grabung, Frankfurt, Franz Steiner Verlag, $221 \mathrm{p}$.

BERTRAN P., ANDRIEUX E., ANTOINE P., COUTARD S., DESCHODT L., GARDĖRE P., HERNANDEZ M., LEGENTIL C., LENOBLE A., LIARD M. 2013 - Distribution and chronology of Pleistocene permafrost features in France: database and first results, Boreas, 43, 3, p. 699-711.

BERTRAN P., LENOBLE A., TODISCO D., DESROSIERS P.M., SØRENSEN M. 2012 - Particle size distribution of lithic assemblages and taphonomy of Palaeolithic sites, Journal of Archaeological Science, 39, 10, p. 3148-3166.

BINFORD L.R. 1983 - In Pursuit of the Past, London, Thames and Hudson, 1983, 256 p. 
BON F. 2002 - L’Aurignacien entre Mer et Océan : Réflexion sur l'unité des phases anciennes de l'Aurignacien dans le sud de la France, Société Préhistorique Française (Mémoire XXIX), Paris, $253 \mathrm{p}$.

BON F. 2005 - Little big tool. Enquête autour du succès de la lamelle, In : F. Le Brun-Ricalens, F. Bon, et J.-G. Bordes (eds), Productions lamellaires attribuées à l'Aurignacien : Chaînes Opératoires et Perspectives Technoculturelles. XIVe congès de l'UISPP, Liège 2-8 Septembre 2001, Luxembourg, Musée national d'Histoire et d'Art (ArchéoLogiques ; 1), p. 479-484.

BON F., MENSAN R., ARAUJO IGREJA M. (De), COSTAMAGNO S., GARDĖRE P., MÉNARD C., SELLAMI F., SZMIDT C., THÉRY-PARISOT I. 2007 - Le site de plein air de Régismont-le-Haut : une halte aurignacienne dans les plaines du Languedoc, In : Qui est l'Aurignacien ?, Aurignac, Editions Musée-forum, cahier 3, p. 53-71.

BON F., SIMONNET R., VÉZIAN J. 2005 - L'équipement lithique des Aurignaciens à la Tuto de Camalhot (SaintJean-de-Verges, Ariège). Sa relation avec la mobilité des groupes et la répartition de leurs activités dans un territoire, In : J. Jaubert et M. Barbaza (eds), Territoires, déplacements, mobilité, échanges durant la Préhistoire. Terres et Hommes du Sud, Paris, Éditions du CTHS, p. 173-184.

BORDES J.-G. 2002 - Les interstratifications Châtelperronien / Aurignacien du Roc-de-Combe et du Piage (Lot, France) : analyse taphonomique des industries lithiques ; implications archéologiques, Université de Bordeaux I, 2002, 365 p., Thèse de doctorat.

BORDES J.-G., LENOBLE A. 2001 - Caminade (Sarlat, Dordogne) (Document final de synthèse de fouilles programmées). Service régional de l'Archéologie Aquitaine, $78 \mathrm{p}$.

BORDES J.-G., LENOBLE A. 2002 - La « lamelle Caminade » : un nouvel outil lithique aurignacien ? Bulletin de la Société préhistorique française, 99, 4, p. 735-749.

BORDES J.-G., BON F., LE BRUN-RICALENS F. 2005 Le transport des matières premières lithiques à l'Aurignacien entre le nord et le sud de l'Aquitaine : faits attendues, faits nouveaux, In : J. Jaubert et M. Barbaza (eds), Territoires, déplacements, mobilité, échanges durant la Préhistoire. Terres et Hommes du Sud, Paris, Éditions du CTHS, p.185-198.

BOSINSKI G., GUICHARNAUD R. 2008 - The working of quartz at the Magdalenian site of Mirande, comm. Nègrepelisse (Tarn-et-Garonne, France), In : Z. Sulgostowska et A. J. Tomaszewski (eds), Man - Millennia - Environment. Studies in honour of Professor Romuald Schild, Warsaw, Institute of Archaeology and Ethnology Polish Academy of Sciences, p. 253-262.
CAPDEVILLE J., TURQ A. 1999 - Notice explicative de la carte géologique de France (1/50000), feuille de Villeneuve-sur-Lot (878), Orléans, BRGM éditions, 1999 , $56 \mathrm{p}$.

CAUX S. 2015 - Du territoire d'approvisionnement au territoire culturel. Pétroarchéologie et techno-économie du silex Grain de mil durant l'Aurignacien dans le Sud-ouest de la France, Université de Bordeaux I, 2015, 247 p., Thèse de doctorat.

CHADELLE J.-P. 1991 - Champ-Parel, Corbiac-Vignoble (Locus 3), Gallia Informations. Préhistoire et Histoire, 1, p. 10.

CHADELLE J.-P. 2000 - Le gisement de Champ-Parel 3 à Bergerac (Dordogne, France). Observations taphonomiques sur un atelier de taille aurignacien en pleinair, Paleo, 12, p. 409-412.

CHADELLE J.-P. 2005 - Productions « intriquées » de lames et de lamelles dans l'Aurignacien de Champ-Parel locus 3 (Bergerac, Dordogne), In : F. Le Brun-Ricalens, F. Bon, et J.-G. Bordes (eds), Productions lamellaires attribuées à l'Aurignacien : Chaînes Opératoires et Perspectives Technoculturelles. XIV congès de l'UISPP, Liège 2-8 Septembre 2001, Luxembourg, Musée national d'Histoire et d'Art (ArchéoLogiques ; 1), p. 193-208.

CHESNAUX L. 2013 - Les microlithes du 62 rue HenryFarman à Paris (15e arrondissement): des flèches diverses pour différents gibiers abattus en des lieux distincts, In : B. Valentin, B. Souffi, T. Ducrocq, J.-P. Fagnart, F. Séara, et C. Verjux (eds), Actes de la table ronde internationale de Paris, 26-27 novembre 2010, Palethnographie du Mésolithique. Recherche sur les habitats de plein air entre Loire et Neckar, Paris, Société Préhistorique Française (Séances de la Société Préhistorique Française 2-1), p. 119-132.

CHESNAUX L. 2014 - Réflexions sur le microlithisme en France au cours du Premier Mésolithique ( $X^{e}$ VIIle millénaire av. JC). Approche technologique, expérimentale et fonctionnelle., Université de Paris I, 2014, 197 p., Thèse de doctorat.

COUTARD J.-P. 2002 - Périglaciaire et expérimentation, Environnements périglaciaires, Notes et comptes-rendus $d u$ groupe de régionalisation du périglaciaire, 27, 9, p. 5-11.

CLAUD E. 2008 - Le statut fonctionnel des bifaces au Paléolithique moyen récent dans le Sud-Ouest de la France. Étude tracéologique intégrée des outillages des sites de La Graulet, La Conne de Bergerac, Combe Brune 2, Fonseigner et Chez-Pinaud / Jonzac, Université de Bordeaux I, 2008, 546 p., Thèse de doctorat.

CROMBÉ P., PERDAEN Y., SERGANT J., CASPAR J.-P. 2001 - Wear Analysis on Early Mesolithic Microliths from the Verrebroek Site, East Flanders, Belgium, Journal of Field Archaeology, 28, 3-4, p. 253-269. 
CURRAY J. R. 1956 - Analysis of two-dimensional orientation data. Journal of Geology, 64, p. 117-134.

DEMARS P.-Y. 1980 - Les matières premières siliceuses utilisées au Paléolitique supérieur dans le bassin de Brive, Université de Bordeaux 1, 1980, 173 p. Thèse de doctorat.

DEMARS P.-Y., LAURENT P. 1992 - Types d'outils lithiques du Paléolithique supérieur en Europe, Paris, Presses du CNRS, $180 \mathrm{p}$.

FEDOROFF N., COURTY M.-A. 1987 - Paléosols, In : J.-C. Miskowsky (ed), Géologie de la Préhistoire, Paris, Association pour l'Étude de l'Environnement Géologique de la Préhistoire, p. 251-280.

FERNANDES P. 2012 - Itinéraires et transformations du silex : une pétroarchéologie refondée, application au Paléolithique moyen, Université de Bordeaux I, 2012, 623 p., Thèse de doctorat.

FERNANDES P., TALLET P., LEANDRI C., TUFFÉRY C., DAVTIAN G., CAVERNE J.-B., BINDER D., TURQ A., MORALA A., LIAGRE J., GAILLOT S., DESSAINT P., RAYNAL J.-P., CHALARD P., TOMASSO A., PLATEL J.-P. 2013 - Rapport d'activité 2013, PCR Réseau de Lithothèques en Rhône-Alpes, 188 p.

FÉBLOT-AUGUSTINS J. 2008 - Paleolithic Raw Material Provenance Studies, In: D. M. Pearsall (ed), Encyclopedia of Archaeology, New York, Academic Press, p.1187-1198.

FISCHER A., VEMMING HANSEN P., RASMUSSEN P. 1984 - Macro and microwear traces on lithic projectile points. Experimental results and prehistoric examples, Journal of Danish Archaeology, 3, p. 19-46.

GASSIN B. 1996 - Évolution socio-économique dans le Chasséen de la grotte de l'Église supérieure (Var) : apport dedustries lithiques, vol. 17, Paris, CNRS éditions, 1996, $328 \mathrm{p}$.

GENESTE J.-M., PLISSON H. 1986 - Le Solutréen de la grotte de Combe Saunière 1 (Dordogne). Première approche palethnologique, Gallia Préhistoire, 29, 1, p. 9-27.

KEELEY L.H. 1980 - Experimental determination of stone tool uses : a microwear analysis, Chicago, University of Chicago Press, 1980, 226 p.

LE BRUN-RICALENS F. 2005 - Reconnaissance d'un « concept technoculturel » de l'Aurignacien ancien ? Modalités, unités et variabilités des productions lamellaires du site d'Hui (Beauville, Lot-et-Garonne, France) : significations et implications, In : F. Le Brun-Ricalens, F. Bon, et J.-G. Bordes (eds), Productions lamellaires attribuées à l'Aurignacien : Chaînes Opératoires et Perspectives Technoculturelles. XIVe congès de I'UISPP, Liège 2-8 Septembre 2001, Luxembourg, Musée national d'Histoire et d'Art (ArchéoLogiques ; 1), p. 157-190.
LUCAS G. 1999 - Production expérimentale de lamelles torses : approche préliminaire, Bulletin de la Société préhistorique française, 96, 2, p. 145-152.

LUCAS G. 2000 - Les industries du Flageolet I (Dordogne) : approche économique technologique, fonctionnelle et analyse spatiale, Université de Bordeaux 1, 2000, 598 p., Thèse de doctorat.

MAURIN G. 2006 - Régismont-le-Haut : découverte et fouille, In : G. Laplace, I. Barandiarán Maestu, A. Saenz de Buruaga, et J. Altuna Echave (eds), Les Aurignaciens pyrénéen des Abeilles et méditerranéen de Régismont-leHaut. Analyses typologique et paléontologique, Les Eyziesde-Tayac, SAMRA, p. 63-83.

MICHEL A. 2010 - L'Aurignacien récent (post-ancien) dans le Sud-Ouest de la France : variabilité des productions lithiques. Révision taphonomique et techno-économique des sites de Caminade-Est, abri Pataud, Roc-de-Combe, Le Flageolet I, La Ferrassie et Combemenue., Université de Bodeaux 1, 2010, 600 p., Thèse de doctorat.

MORALA A. 1980 - Observations sur le Périgordien, l'Aurignacien et leurs matières premières lithiques en HautAgenais, Mémoire de l'École des Hautes Études en Sciences sociales, Toulouse, $190 \mathrm{p}$.

MORALA A. 1984 - Périgordien et Aurignacien en HautAgenais: étude d'ensembles lithiques, Archives d'Écologie Préhistorique, Toulouse, 7, $140 \mathrm{p}$.

MORALA A., LENOIR M., TURQ A. 2005 - Production et utilisation de supports normalises lamino-lamellaires dans les chaînes opératoires des grattoirs Caminade du site Pigeonnier à Gensac (Gironde, France), in F. Le BrunRicalens, F. Bon, et J.-G. Bordes (eds), Productions lamellaires attribuées à l'Aurignacien : Chaînes Opératoires et Perspectives Technoculturelles. XIV congès de l'UISPP, Liège 2-8 Septembre 2001, Luxembourg, Musée national d'Histoire et d'Art (ArchéoLogiques ; 1), p. 257-271.

MOSS E.H., NEWCOMER M.H. 1982 - Reconstruction of tool use at Pincevent : microwear and experiments, In : D. Cahen (ed), Tailler ! pour quoi faire : Préhistoire et technologie lithique II, Recent progress in microwear studies, Tervuren, Musée royal de l'Afrique centrale, p. 289-312.

MURRAY A.S., WINTLE A.G. 2000 - Luminescence dating of quartz using an improved single-aliquot regenerativedose protocol, Radiation measurements, 32, 1, p. 57-73.

NORMAND C., O'FARRELL M., RIOS GARAIZAR J. 2008 - Quelle(s) utilisation(s) pour les productions lamellaires de l'Aurignacian archaïque ? Quelques données et réflexions à partir des exemplaires de la Grotte d'Isturitz (Pyrénées-Atlantiques ; France), P@lethnologie: Revue bilingue de Préhistoire, 1, p. 7-46.

O'FARRELL M. 2004 - Les pointes de La Gravette de Corbiac (Dordogne) et considérations sur la chasse au Paléolithique supérieur ancien, In : P. Bodu et 
C. Constantin (eds), Approches fonctionnelles en préhistoire, $X X V^{e}$ Congrès préhistorique de France, Nanterre, 24-26 novembre 2000, Paris, Société préhistorique française, p. 121-138.

O'FARRELL M. 2005 - Étude préliminaire des éléments d'armature lithique de l'Aurignacien ancien de Brassempouy, In : F. Le Brun-Ricalens, F. Bon, et J.-G. Bordes (eds), Productions lamellaires attribuées à l'Aurignacien : Chaînes Opératoires et Perspectives Technoculturelles. XIVe congès de I'UISPP, Liège 2-8 Septembre 2001, Luxembourg, Musée national d'Histoire et d'Art (ArchéoLogiques ; 1), p. 395-412.

ORTEGA CORDELLAT I., RIOS GARAIZAR J., IBÁÑEZ ESTÉVEZ J.J., GONZÁLEZ URQUIJO J.E., BOËDA É., SELLAMI F. 2006 - L'occupation de l'Aurignacien ancien de Barbas III (Creysse, Dordogne) : résultats préliminaires sur la fonction du site, PALEO, 18, p. 115-142.

PELEGRIN J. 1991 - Les savoir-faire: une très longue histoire. Terrain : Carnets du Patrimoine ethnologique, 16, p.106-113.

PELEGRIN J. 2000 - Les techniques de débitage laminaire au Tardiglaciaire: critères de diagnose et quelques réflexions, In : B. Valentin, $\mathrm{P}$. Bodu, et $\mathrm{M}$. Christensen dir, L'Europe Centrale Et Septentrionale Au Tardiglaciaire: Actes de la Table-ronde Internationale de Nemours, 14-1516 Mai 1997, Nemours, Association pour la Promotion de la Recherche archéologique en lle-de-France, p. 73-86.

PERLÈS C. 1991 - Économie des matières premières et économie du débitage : deux conceptions opposées ? in 25 Ans d'études technologiques en préhistoire. $\mathrm{X}^{\mathrm{e}}$ Rencontres Internationales d'archéologie et d'histoire d'Antibes, Juan-les-Pins, Éditions APDCA, p. 35-45.

PESESSE D., MICHEL A. 2006 - Le burin des Vachons : apports d'une relecture technologique à la compréhension de l'Aurignacien récent du nord de l'Aquitaine et des Charentes, Paleo, 18, p. 143-160.

PESESSE D. 2008 - Les premières sociétés gravettiennes : analyse comparée des systèmes lithiques de la fin de l'Aurignacien aux débuts du Gravettien, Université de Provence, 2008, 2 volumes, 460 p., Thèse de doctorat.

PÉTILLON J.-M., BIGNON O., BODU P., CATTELAIN P., DEBOUT G., LANGLAIS M., LAROULANDIE V., PLISSON H., VALENTIN B. 2011 - Hard core and cutting edge: experimental manufacture and use of Magdalenian composite projectile tips, Journal of Archaeological Science, 38, 6, p. 1266-1283.

PHILIBERT S. 2002 - Les derniers «Sauvages». Territoires économiques et systèmes techno-fonctionnels mésolithiques, Oxford, BAR International Series 1069, 193.

PICAVET R., CHESNAUX L., FERNANDES P., KAWALEC E., MORALA M., RUÉ M., TALLET P. 2013 - La station aurignacienne de Brignol à Villeneuve-sur-Lot (47). Rapport final d'opération, 2013, 358 p.

PLISSON H. 1985 - Étude fonctionnelle d'outillages lithiques préhistoriques par l'analyse des micro-usures: recherche méthodologique et archéologique, Université de Paris I, 1985, 403 p., Thèse de doctorat.

QUINTARD A. 1995 - Monsempron, Las Pélénos, Bilan Scientifique Régional 1994, SRA Aquitaine, p. 91-92.

RASMUSSEN S. O., BIGLER M., BLOCKLEY S. P., BLUNIER T., BUCHARDT S. L., CLAUSEN H. B., CVIJANOVIC I., DAHL-JENSEN D., JOHNSEN S. J., FISCHER H., GKINIS V., GUILLEVIC M., HOEK W. Z., LOWE J., PEDRO J. B., POPP T., SEIERSTAD I. K., STEFFENSEN J. P., SVENSSON A. M., VALLELONGA P., VINTHER B. M., WALKER M. J. C., WHEATLEY J. J., WINSTRUP M. 2014 - Stratigraphic framework for abrupt climatic changes during the Last Glacial period based on three synchronized greenland ice-core records: refining and extending the INTIMATE event stratigraphy. Quaternary Science Reviews, 106, p. 14-28.

RIGAUD J.-Ph. 1982 - Le Paléolithique en Périgord: les données du sud-ouest sarladais et leurs implications, Université de Bordeaux I, 1982, 494 p., Thèse de doctorat.

RIGAUD J.-Ph. 2001 - À propos de la contemporanéité du Castelperronien et de l'Aurignacien ancien dans le nord-est de l'Aquitaine : une révision des données et ses implications, In : J. Zilhao, T. Aubry, A. Faustino Carvalho (Eds.), Les premiers hommes modernes de la péninsule Ibérique, Actes de la commission VIII de l'UISPP, Vila Nova de Foz Côa, 22-24 octobre 1998, Trabalhos de Arqueologia, vol. 17, p. 61-68.

SEMENOV S.A. 1964 - Prehistoric technology, an experimental study of the oldest tools and artefacts from traces of manufacture and wear, Londres, Cory, Adams and Mackay, $211 \mathrm{p}$.

SONNEVILLE-BORDES D. (de), MORTUREUX B. 1956 Outils aurignaciens nouveaux ou rares, L'Anthropologie, 60 , p. 574-578.

SONNEVILLE-BORDES D.(de) 1960 - Le Paléolithique supérieur en Périgord, Bordeaux, Delmas, 580 p.

SONNEVILLE-BORDES D. (de) 1970 - Les industries aurignaciennes de l'abri de Caminade-Est, commune de La Canéda (Dordogne), Quaternaria, Roma, 13, p. 77-131.

SONNEVILLE-BORDES D. (de), MORTUREUX B. 1955 L'abri Caminade, commune de La Canéda (Dordogne), Bulletin de la Société préhistorique française, 52, 9, p. 608-619.

SONNEVILLE-BORDES D. (de), MORTUREUX B. 1956 Outils aurignaciens nouveaux ou rares, L'Anthropologie, 60 , p. $574-578$. 
TARTAR É., TEYSSANDIER N., BON F., LIOLIOS D. 2006 - Équipement de chasse, équipement domestique: une distinction efficace? Réflexion sur la notion d'investissement technique dans les industries aurignaciennes, in L. Astruc, F. Bon, V. Léa, P.-Y. Milcent, et S. Philibert (eds), Normes Techniques et Pratiques Sociales. De la Simplicité des Outillages Pré- et Protohistorique, $X X V{ }^{*}$ rencontres internationales d'archéologie et d'histoire d'Antibes, Antibes, Éditions APDCA, p. 109-117.

TIXIER J. 1991 - Champ-Parel, Corbiac-Vignoble 2 (CV2), Gallia Informations. Préhistoire et Histoire, 1, p. 8-10.

TIXIER J., REDURON M. 1991 - Et passez au pays des silex : rapportez-nous des lames ! In : 25 Ans d'études technologiques en préhistoire. $X{ }^{*}$ Rencontres Internationales d'archéologie et d'histoire d'Antibes, Juanles-Pins, Éditions APDCA Juan-les-Pins, Éditions APDCA, p. 237-243.

TODISCO D., BHIRY N., DESROSIERS P.M. 2009 Paleoeskimo site taphonomy: An assessment of the integrity of the Tayara site, Qikirtaq Island, Nunavik, Canada, Geoarchaeology, 24, 6, p. 743-791.
TURQ A. 1992 - Le Paléolithique inférieur et moyen entre les vallées de la Dordogne et du Lot. Thèse de doctorat. Université Bordeaux 1, $782 \mathrm{p}$.

TURQ A. 2000 - Le Paléolithique inférieur et moyen entre Dordogne et Lot. Paleo, supplément $n^{\circ} 2,456 \mathrm{p}$.

VALENTIN B., WEBER M.J., BODU P. 2014 - Initialisation and progression of the core reduction process at Donnemarie-Dontilly (Seine-et-Marne, France), site of the Belloisian tradition : new interprative key for comparisons with contemporaneous industries and FedermesserGruppen assemblages, Bulletin de la Société préhistorique française, 111, 4, p. 659-678.

VALLIN L., CASPAR J.-P., GUILLEMET G., MASSON B., OZOUF J.-C. 2013 - Altérations des artefacts préhistoriques en silex par les processus périglaciaires : présentation des expériences conduites au Centre de Géomorphologie du CNRS de Caen, Quaternaire, 24, 3, p. 259-266.

VÉZIAN JOSEPH, VÉZIAN JEAN. 1966 - Les gisements de la grotte de Saint-Jean-de-Verges (Ariège), Gallia Préhistoire, 9, 1, p. 93-130. 